\title{
Design, synthesis and antibacterial activity of a novel alkylide: 3-O-(3-aryl-propenyl)clarithromycin derivatives
}

\author{
Jian-Hua Liang ${ }^{1}$, Yue-Ying Wang ${ }^{1}$, Dan-Yang Zhu ${ }^{1}$, Li-Jing Dong ${ }^{1}$, Mao-Mao An ${ }^{2}$, Rui Wang ${ }^{2}$ and \\ Guo-Wei Yao ${ }^{1}$ \\ A series of novel 3-0-(3-aryl-propenyl)clarithromycin derivatives were designed, synthesized and evaluated for their in vitro \\ antibacterial activities. Regioselective allylation at $3-\mathrm{OH}$ was efficiently achieved in the presence of 9-oxime ether, compared \\ with 9-keto. Most of the side chains were identified as the 3-0-(3-aryl-Z-prop-1-enyl) group, not the expected 3-0-(3-aryl- \\ E-prop-2-enyl) group. Some derivatives of this series showed improved activities against erythromycin-resistant Staphylococcus \\ aureus and Staphylococcus pneumoniae compared with the reference compound, clarithromycin, but weaker activities against \\ susceptible strains.
}

The Journal of Antibiotics (2009) 62, 605-611; doi:10.1038/ja.2009.89; published online 28 August 2009

Keywords: allyl; erythromycin; heck; isomerization; regioselectivity

\section{INTRODUCTION}

Erythromycin, a 14-membered macrolide antibiotic, has been used safely and effectively against respiratory tract infections since 1952. However, owing to its acid-instability, clarithromycin (6-O-methylerythromycin) as the second-generation erythromycin was developed to address the problem of ketalization of the C-9 carbonyl group by the 6-OH group. ${ }^{1}$ It is noted that regioselective methylation at $6-\mathrm{OH}$ to yield clarithromycin was not achieved until the introduction of oxime ether in the 9-carbonyl group. ${ }^{2}$ Similarly, new emerging cethromycin [3-oxo-6-O-(3-(3'-quinolyl)-2-propenyl)erythromycin derivative] as the third-generation erythromycin was synthesized by regioselective allylation at $6-\mathrm{OH}^{3-5}$ to overcome the relatively recent issues associated with the development of bacterial resistance.

Most of the third-generation erythromycin, cethromycin included, shares two distinguishing structural features: first, cladinose is not an essential moiety for the antibacterial activity, and removal of cladinose can prevent the induction of resistance (Figure 1). As a result, modification at $3-\mathrm{OH}$ led to the emergence of ketolide, ${ }^{3-6}$ acylide, ${ }^{7}$ bicyclolide, ${ }^{8}$ anhydrolide, ${ }^{9} 2,3$-enol-ether, ${ }^{10} 3$-deoxy, ${ }^{11} 3$-ether, ${ }^{12} 3,6$ ketal $^{13}$ and 3,6-ether. ${ }^{14}$ Second, an aryl side chain tethered to the erythronolide core is believed to be very important for the improvement of activities against erythromycin-resistant bacteria, due to the interaction with a secondary ribosomal binding site. ${ }^{15}$

The results published were not ours. In the search for novel erythromycin derivatives against erythromycin-resistant bacteria, the allyl group was a very important one for various further modifications at 6-OH of erythromycin. ${ }^{4,5}$ To the best of our knowledge, the introduction of the allyl group at 3-OH of 14-membered macrolides was not disclosed yet, in addition to the recent modification of 16-membered macrolides, leucomycin. ${ }^{16}$

\section{RESULTS AND DISCUSSION}

Initially, we attempted to attach an allyl group to $3-\mathrm{OH}$ of the $2^{\prime}-\mathrm{O}$ Ac-3-OH-6-O-methylerythromycin 11, 12-carbonate with the structure of 9-keto, but unfortunately it failed and the major product in the resulting mixture was identified as 10,11-anhydro-3-OH-6-O-methylerythromycin A after methanolysis. Later, we found that the introduction of 9-oxime ether could dramatically improve the selectivity of allylation at 3-OH. Thus, a facile synthesis of 3-O-allyl of clarithromycin was conducted as a lead compound. In this study, we report a novel series of macrolides: 3-O-(3-aryl-propenyl)clarithromycin derivatives, which we named alkylides.

The synthesis of the targeted alkylide 9 in five steps from the intermediate 3-OH-6-O-methylerythromycin 9-O-(2-chlorobenzyl) oxime 4 was outlined in Schemes 1 and 2. Compound 4 was obtained by the acidic hydrolysis of $2^{\prime}, 4^{\prime \prime}$-O-bis(trimethylsilyl) 6-O-methylerythromycin 9-O-(2-chlorobenzyl)oxime $3,{ }^{17}$ which was an important intermediate for the synthesis of clarithromycin. Next, acetylation at $2^{\prime}-\mathrm{OH}$ of 4 was followed by carbonation at 11, 12-OH generated 6. Consequently, allylation at $3-\mathrm{OH}$ was efficiently achieved in the presence of allyl bromide and $\mathrm{KOtBu}$, and 7 was produced in high yield (95.2\%). Thus, various hetero-aryl bromides were used to install

${ }^{1}$ School of Life Science, Beijing Institute of Technology, Beijing, China and ²Department of Clinical Pharmacology, Chinese Peoples Liberation Army General Hospital, Beijing, China

Correspondence: Professor J-H Liang, School of Life Science, Beijing Institute of Technology, Zhongguancun South street 5, Beijing 100081, China

E-mail: ljhbit@bit.edu.cn

Received 23 June 2009; revised 20 July 2009; accepted 4 August 2009; published online 28 August 2009 


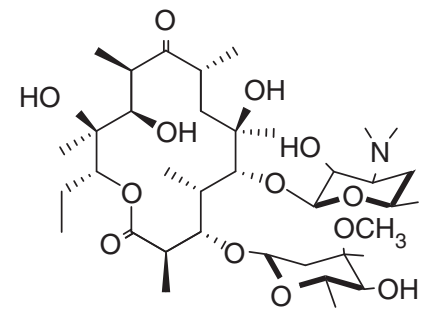

erythromycin

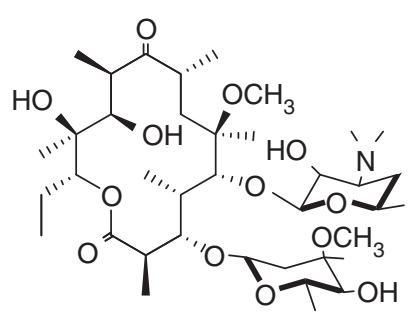

clarithromycin

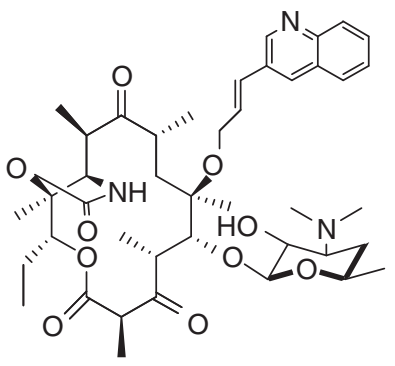

cethromycin

Figure 1 Structure of erythromycin, clarithromycin and cethromycin.

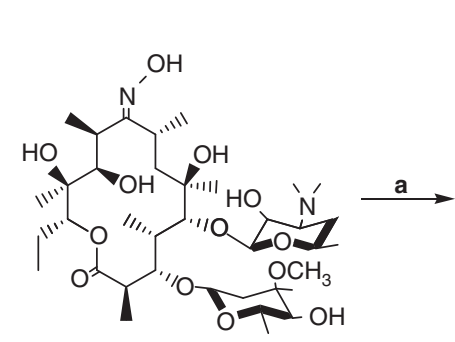

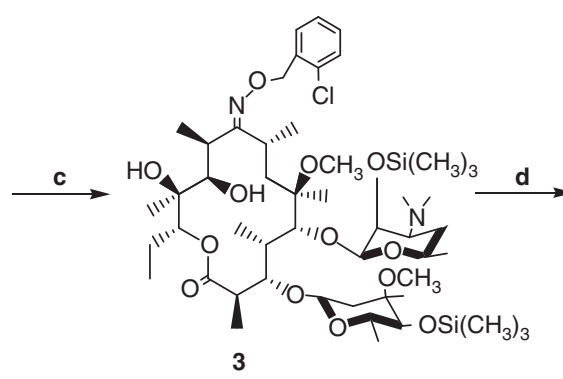

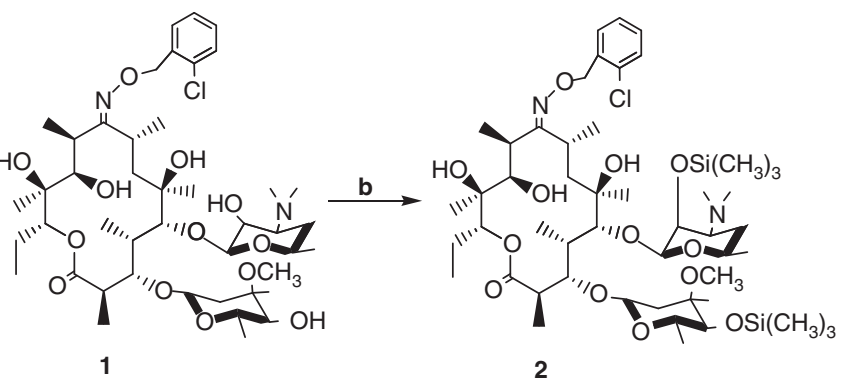

2

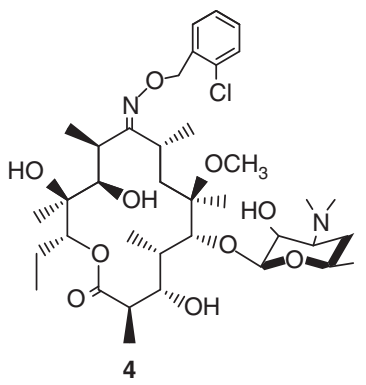

Scheme 1 Reagents and conditions: (a) 2-chlorobenzyl chloride, $\mathrm{KOH}$, acetonitrile, 98.6\%; (b) hexamethyldisilizane (HMDS), pyridine hydrochloride, acetonitrile, $85.8 \%$; (c) $\mathrm{CH}_{3} \mathrm{l}, \mathrm{KOH}$, THF/dimethyl sulfoxide (DMSO), $0{ }^{\circ} \mathrm{C}, 98.6 \%$; (d) $\mathrm{C}_{2} \mathrm{H}_{5} \mathrm{OH} / \mathrm{H}_{2} \mathrm{O}, \mathrm{HCl}, 73.8 \%$.
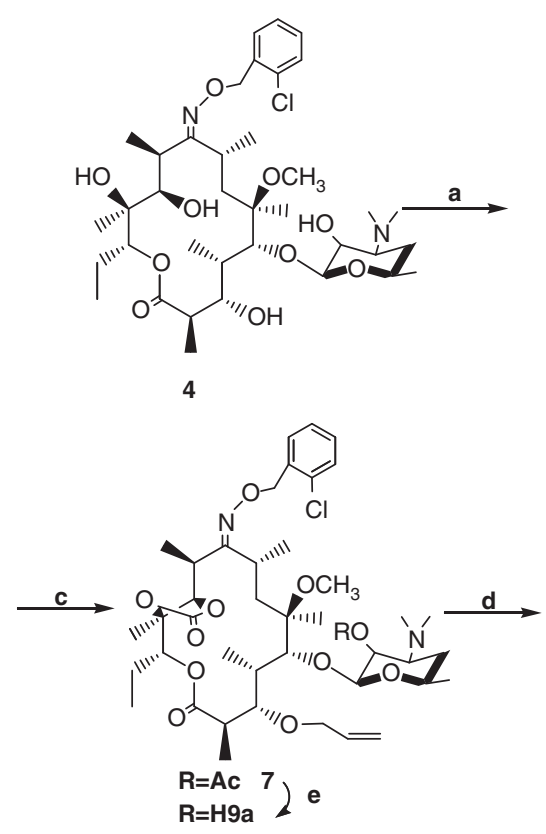
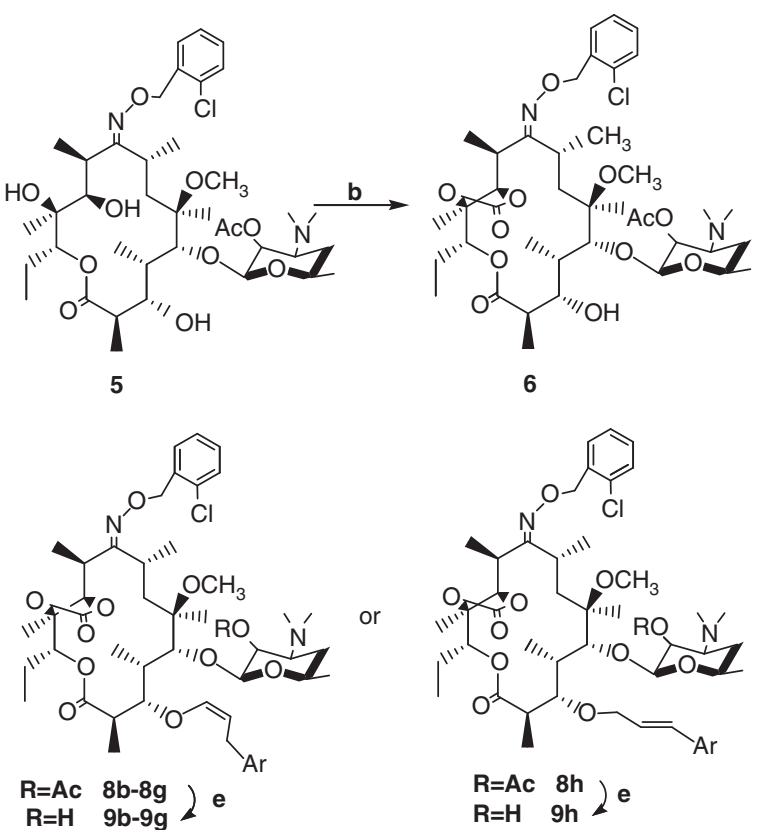

Scheme 2 Reagents and conditions: (a) $\mathrm{Ac}_{2} \mathrm{O}, \mathrm{CH}_{2} \mathrm{Cl}_{2}, 94.5 \%$; (b) bis(trichloromethyl)carbonate (BTC), pyridine, $\mathrm{CH}_{2} \mathrm{Cl}_{2}, 0{ }^{\circ} \mathrm{C}, 98.7 \%$; (c) allyl bromide, $\mathrm{KOtBu}, \mathrm{THF} / \mathrm{DMSO}, 0^{\circ} \mathrm{C}, 95.2 \%$; (d) $\mathrm{Pd}(\mathrm{OAC})_{2}, \mathrm{P}(\mathrm{O}-\mathrm{MePh})_{3}, \mathrm{Et}_{3} \mathrm{~N}$, acetonitrile, hetoro-aryl bromide, $60^{\circ} \mathrm{C} 1 \mathrm{~h}$ then $90^{\circ} \mathrm{C} 24 \mathrm{~h} ;(\mathrm{e}) \mathrm{MeOH}, 60^{\circ} \mathrm{C}$. 


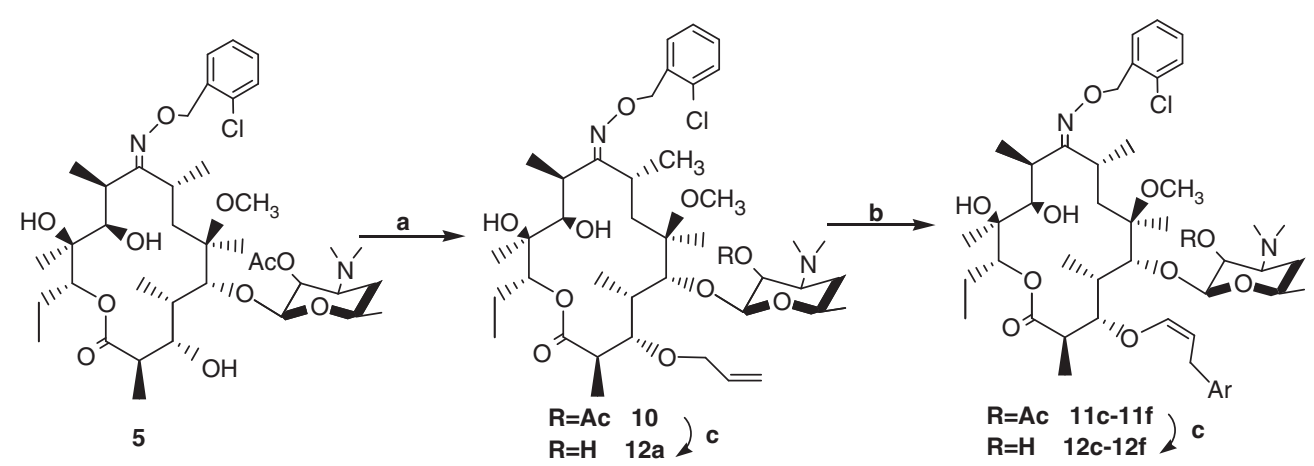

Scheme 3 Reagents and conditions: (a) allyl bromide, KOtBu, THF/DMSO, $0^{\circ} \mathrm{C}, 85.7 \%$; (b) $\mathrm{Pd}(\mathrm{OAc})_{2}, \mathrm{P}(\mathrm{O}-\mathrm{MePh})_{3}$, Et $3 \mathrm{~N}^{\mathrm{N}}$, acetonitrile, hetoro-aryl bromide, $60^{\circ} \mathrm{C} 1 \mathrm{~h}$ then $90^{\circ} \mathrm{C} 24 \mathrm{~h}$; (c) $\mathrm{MeOH}, 60^{\circ} \mathrm{C}$.

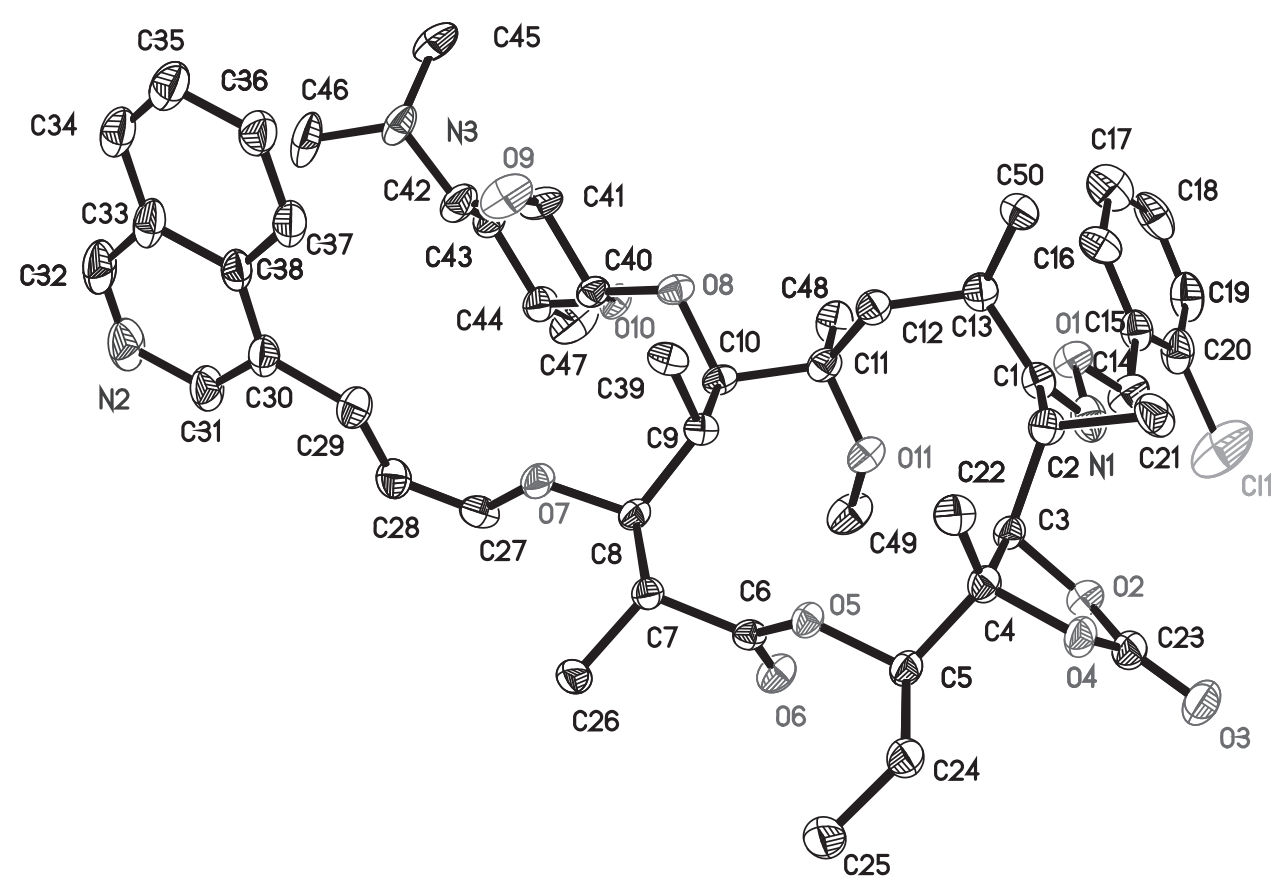

Figure 2 Crystal structure of $9 f$ (4-isoquinolyl).

the aryl side chain by Heck reaction, catalyzed by $\mathrm{Pd}(\mathrm{OAc})_{2} / \mathrm{P}(o-$ $\mathrm{MePh})_{3}$. The major products of Heck reaction and its methanolysis were purified to yield $\mathbf{8}$ and $\mathbf{9}$ by column chromatography using $\mathrm{CH}_{2} \mathrm{Cl}_{2} / \mathrm{EtOH} / \mathrm{NH}_{3} \cdot \mathrm{H}_{2} \mathrm{O}$ (15:0.4:0.1) and petroleum ether/acetone/ triethylamine (5:5:0.2) as an eluent, respectively.

To confirm the regioselectivity of 3-OH, compound 12 was synthesized without the protection of $11,12-\mathrm{OH}$ (Scheme 3 ). The results proved that the regioselective allylation of $\mathbf{5}$ was also conducted in good yield $(85.7 \%)$ and, as indicated, the regioselectivity at 3-OH was mainly influenced by 9 -oxime ether.

A combination of ${ }^{1} \mathrm{H}$ NMR, COSY, ${ }^{13} \mathrm{C}$ NMR and HRMS was used to analyze the structure of this series. Surprisingly, the NMR spectrum showed that the aryl side chains of $\mathbf{9 b}-\mathbf{9 g}$ and $\mathbf{1 2 c - 1 2 f}$ were characterized by predominant $Z$ configuration $(J=6 \mathrm{~Hz})$, such as the 1-phenyl (a mixture of Z/E: 10:4), 3-pyridyl, 5-pyrimidyl, 3-quinolyl, 4-isoquinolyl and 5-isoquinolyl groups, whereas the 5 -indolyl $\mathbf{9 h}$ side chain had $E$ configuration $(J=16 \mathrm{~Hz})$. This was significantly different from the previous results of allylation at $6-\mathrm{OH}^{4}$ Further study by $\mathrm{X}$-ray crystallography of $\mathbf{9 f}$ disclosed that the allylic double bond isomerized from 2-position to 1-position, and the Heck-isomerization to furnish enol concomitantly resulted in $Z$ configuration, as shown in Figure 2. Therefore, with the exception of $\mathbf{9 h}$, the real structure of $\mathbf{9 b}$ $\mathbf{9 g}$ and $12 \mathrm{c}-12 \mathrm{f}$ should be the 3-O-(3-aryl-Z-prop-1-enyl)clarithromycin derivatives, not the expected 3-O-(3-aryl-E-prop-2-enyl)clarithromycin derivatives.

The in vitro antibacterial activity of alkylides 9 and 12 was assessed against erythromycin-susceptible and erythromycin-resistant bacteria, including Staphylococcus aureus and Streptococcus pneumoniae. Data are represented in Table 1 as the minimal inhibitory concentration, which is determined by the broth microdilution method as recommended by the NCCLS (National Committee of Clinical Laboratory Standard). ${ }^{18}$

Some of the alkylides such as 9, 12 showed improved activities compared with the reference compound, clarithromycin, against resistant pathogens, particularly for $S$. aureus. Among them, 9d and 12d (3-pyrimidinyl) were promising candidates. The compounds with the mono-heteroaryl ring $(\mathbf{9 c}-\mathbf{9 d}, \mathbf{1 2 c}-\mathbf{1 2 d})$ and the parent compounds $9 \mathbf{a}$ and $12 \mathbf{a}(\mathrm{H})$ possessed better activity against resistant pathogens than did those with fused aryl rings (9e-9h, 12e-12f). 
Table 1 Antibacterial effects of alkylides against selected respiratory tract pathogens

In vitro $M I C(\mu g / m l)$

\begin{tabular}{|c|c|c|c|c|c|c|c|c|c|c|c|}
\hline \multicolumn{3}{|c|}{ Compound } & \multicolumn{4}{|c|}{ Staphylococcus aureus } & \multicolumn{5}{|c|}{ Staphylococcus pneumoniae } \\
\hline & Aryl & Config. & $\operatorname{ATCC} 29213^{\mathrm{a}}$ & $D 18^{\mathrm{b}}$ & $D 6^{\mathrm{b}}$ & $D 9^{\mathrm{b}}$ & ATCC $49619^{a}$ & SPJ $8^{\mathrm{b}}$ & $S P J 15^{b}$ & $C P 18^{\mathrm{b}}$ & $S P M 12^{\mathrm{b}}$ \\
\hline $9 a$ & $\mathrm{H}$ & - & 8 & 16 & 16 & 16 & 4 & $>16$ & 16 & $>16$ & $>16$ \\
\hline $9 b$ & 1-phenyl & ZIE & 64 & 64 & $>64$ & 64 & 8 & $>16$ & 16 & $>16$ & $>16$ \\
\hline $9 c$ & 3-pyridyl & $z$ & 8 & 32 & 32 & 16 & 1 & $>16$ & $>16$ & $>16$ & $>16$ \\
\hline $9 f$ & 4-isoquinolyl & $z$ & $>64$ & 64 & 64 & $>64$ & 4 & $>16$ & 16 & $>16$ & $>16$ \\
\hline 9g & 5-isoquinolyl & $z$ & $>64$ & $>64$ & $>64$ & $>64$ & 4 & $>16$ & 8 & $>16$ & $>16$ \\
\hline $9 h$ & 5-indolyl & $E$ & $>64$ & $>64$ & $>64$ & $>64$ & 8 & $>16$ & 16 & $>16$ & $>16$ \\
\hline $12 \mathrm{a}$ & $\mathrm{H}$ & - & 32 & 16 & 64 & 16 & 16 & $>16$ & $>16$ & $>16$ & $>16$ \\
\hline $12 c$ & 3-pyridyl & $z$ & 32 & 16 & 32 & 16 & 4 & $>16$ & $>16$ & $>16$ & $>16$ \\
\hline $12 d$ & 5-pyrimidyl & $Z$ & 8 & 16 & 32 & 16 & 1 & $>16$ & $>16$ & $>16$ & $>16$ \\
\hline
\end{tabular}

Abbreviations: Config., configuration; MIC, minimal inhibitory concentration.

aErythromycin-susceptible.

bErythromycin-resistant.

The crystal structure of 9 f (4-isoquinolyl) disclosed that the fused aryl rings, regardless of the location of the nitrogen $(\mathbf{e}-\mathbf{g})$, may sterically prevent the $3^{\prime}$-dimethylamino group from approaching the binding site, resulting in a decrease in activities. Compounds 9c, 12c (3-pyridyl) and 9d, 12d (3-pyrimidinyl) showed better activities than did the parent compounds $9 \mathbf{a}$ and $12 \mathbf{a}(\mathrm{H})$ against erythromycin-susceptible pathogens, but unfortunately no significant difference was found between them against erythromycin-resistant pathogens.

In conclusion, the 3-O-allyl clarithromycin derivative was synthesized in high regioselectivity to serve as a template. The introduction of the 3-O-aryl-propenyl group led to the acquisition of activities against erythromycin-resistant pathogens than reference compound, clarithromycin. The further chemical modification to improve activities is ongoing.

\section{EXPERIMENTAL SECTION}

All solvents and reagents were obtained from commercial sources and used without further purification unless otherwise noted. Column chromatography was performed on silica gel (200-300 mesh for 8 and 300-400 mesh for 9). ${ }^{1} \mathrm{H}$ and ${ }^{13} \mathrm{C}$ NMR spectra were recorded in $\mathrm{CDCl}_{3}$ on a Bruker ARX 400 and $600 \mathrm{MHz}$ (Bruker BioSpin AG Ltd, Beijing, China) with tetramethylsilane (TMS) as an internal standard. The assignments of 4, 7, 9c and 12a were made on the basis of $\mathrm{D}_{2} \mathrm{O}$ exchange and ${ }^{1} \mathrm{H}_{-}{ }^{1} \mathrm{H}$ COSY. HRMS were obtained with Bruker Apex II ICRMS, or Bruker Apex IV FTMS.

\section{Erythromycin A 9-O-(2-chlorobenzyl)oxime (1)}

To a solution of Erythromycin A, 9-O-oxime $(10 \mathrm{~g}, 13.3 \mathrm{mmol})$ in acetonitrile $(120 \mathrm{ml})$ was added 2-chlorobenzyl chloride $(2.1 \mathrm{ml}, 16.5 \mathrm{mmol})$ and $\mathrm{KOH}$ $(1.3 \mathrm{~g}, 21 \mathrm{mmol})$. The reaction mixture was stirred at room temperature for $2 \mathrm{~h}$, and in ice bath for another $1 \mathrm{~h}$. The resulting precipitate was filtrated and dried to yield $\mathbf{1}(11.5 \mathrm{~g}, 98.6 \%)$.

\section{2, 4"-O-bis(trimethylsilyl)erythromycin A 9-O-(2-chlorobenzyl)oxime (2)}

To a solution of $\mathbf{1}(23 \mathrm{~g}, 26.3 \mathrm{mmol})$ in acetonitrile $(200 \mathrm{ml})$ was added pyridine hydrochloride $(8.8 \mathrm{~g}, 76.1 \mathrm{mmol})$ and hexamethyldisilizane (HMDS) $(20 \mathrm{ml}$, $94.2 \mathrm{mmol})$. The reaction mixture was stirred at room temperature for $1 \mathrm{~h}$ and extracted twice with petroleum ether. The organic phase was dried over $\mathrm{MgSO}_{4}$ and evaporated to yield $2(23.0 \mathrm{~g}, 85.8 \%) \cdot{ }^{13} \mathrm{C} \mathrm{NMR}\left(100 \mathrm{MHz}, \mathrm{CDCl}_{3}\right) \delta: 175.8$, $172.0,135.4,133.0,129.5,129.2,128.8,126.8,102.5,96.5,81.4,80.8,79.3,76.9$, $75.3,74.2,73.2,73.1,72.8,70.4,67.6,65.1,64.9,49.6,44.6,40.9,40.2,38.4,35.8$, $33.2,29.6,26.8,26.7,22.1,21.7,21.2,19.3,18.5,16.2,15.6,14.3,10.7,9.6,0.9,0.8$.

\section{$2^{\prime}, 4^{\prime \prime}$-O-bis(trimethylsilyl)-6-O-methylerythromycin A 9-O-(2-chlorobenzyl)oxime (3)}

To an ice-cold solution of $2(23.0 \mathrm{~g}, 22.6 \mathrm{mmol})$ in $80 \mathrm{ml}$ THF and $80 \mathrm{ml}$ dimethyl sulfoxide (DMSO) was added $\mathrm{CH}_{3} \mathrm{I}(2.4 \mathrm{ml}, 47.3 \mathrm{mmol})$ and $\mathrm{KOH}$ $(2.4 \mathrm{~g}, 42.8 \mathrm{mmol})$. The reaction mixture was stirred at $0^{\circ} \mathrm{C}$ for $1 \mathrm{~h}$, and then was poured with water and extracted with petroleum ether. The organic layer was concentrated to yield 3 ( $23.0 \mathrm{~g}, 98.6 \%)$.

3-OH-6-O-methylerythromycin A 9-O-(2-chlorobenzyl)oxime (4) To a solution of $\mathbf{3}(23.0 \mathrm{~g}, 22.3 \mathrm{mmol})$ in ethanol $(80 \mathrm{ml})$ was slowly added a solution of diluted $\mathrm{HCl}(8 \mathrm{ml} \mathrm{HCl}$ in $80 \mathrm{ml}$ water $)$ over $10 \mathrm{~min}$. The reaction mixture was stirred at room temperature for $1.5 \mathrm{~h}$ and neutralized with $\mathrm{NH}_{3} \cdot \mathrm{H}_{2} \mathrm{O}(36 \%, 8 \mathrm{ml})$. The precipitate was collected by filtration and washed with water to yield $4(12.0 \mathrm{~g}, 73.8 \%)$. MS(MALDI-TOF) $\left(\mathrm{M}+\mathrm{Na}^{+}\right) \mathrm{m} / z$ 751.5, calcd for $\mathrm{C}_{37} \mathrm{H}_{61} \mathrm{ClN}_{2} \mathrm{O}_{10} 728.4(\mathrm{M}) .{ }^{1} \mathrm{H} \mathrm{NMR}\left(400 \mathrm{MHz}, \mathrm{CDCl}_{3}\right) \delta: 7.41-7.22$ ( $\mathrm{m}, 4 \mathrm{H}$, benzyl ring), $5.22-5.06\left(\mathrm{~m}, 3 \mathrm{H}, \mathrm{H}-13,-\mathrm{CH}_{2}-\mathrm{PhCl}\right), 4.36-4.35(\mathrm{~m}, 2 \mathrm{H}$, $\left.\mathrm{H}-1^{\prime}, 11-\mathrm{OH}\right), 3.81$ (s, 1H, H-11), 3.78-3.71 (m, 1H, H-8), 3.64 (m, 2H, H-5, $\left.2^{\prime}-\mathrm{OH}\right), 3.54-3.51\left(\mathrm{~m}, 2 \mathrm{H}, \mathrm{H}-3, \mathrm{H}-5^{\prime}\right), 3.28(\mathrm{~s}, 12-\mathrm{OH}), 3.23\left(\mathrm{dd}, 1 \mathrm{H}, \mathrm{H}-2^{\prime}\right)$, $2.82\left(\mathrm{~s}, 3 \mathrm{H}, 6-\mathrm{OCH}_{3}\right), 2.66-2.62(\mathrm{~m}, 1 \mathrm{H}, \mathrm{H}-2), 2.57$ (q, 1H, H-10), 2.49-2.43 (m, 1H, H-3'), $2.24\left(\mathrm{~s}, 6 \mathrm{H},-\mathrm{N}\left(\mathrm{CH}_{3}\right)_{2}\right), 2.09(\mathrm{~m}, 1 \mathrm{H}, \mathrm{H}-4), 1.97-19.2(\mathrm{~m}, 1 \mathrm{H}$, H-14eq), 1.67-1.50 (m, 3H, H-4', H-7ax, H-14ax), 1.38 (s, 3H, 6-CH - $_{3}, 1.35-1.22$ (m, 8H, H-7eq, H-4'eq, 5'- $\left.\mathrm{CH}_{3}, 2-\mathrm{CH}_{3}\right), 1.18\left(\mathrm{~s}, 3 \mathrm{H}, 12-\mathrm{CH}_{3}\right), 1.13$ (d, 3H, 10$\left.\mathrm{CH}_{3}\right), 1.07\left(\mathrm{~d}, 3 \mathrm{H}, 4-\mathrm{CH}_{3}\right), 0.97\left(\mathrm{~d}, 3 \mathrm{H}, 8-\mathrm{CH}_{3}\right), 0.83\left(\mathrm{t}, 3 \mathrm{H}, 15-\mathrm{CH}_{3}\right)$. ${ }^{13} \mathrm{C} \operatorname{NMR}\left(100 \mathrm{MHz}, \mathrm{CDCl}_{3}\right) \delta: 174.9,170.5,135.7,133.4,129.8,129.2,128.9$, 126.5, 106.7, 87.9, 78.8, 78.3, 76.8, 73.9, 72.6, 70.6, 70.4, 70.1, 65.6, 49.3, 44.5, $40.2,37.3,35.9,33.1,28.026 .4,21.5,21.2,18.7,18.3,16.2,15.3,15.1,10.4,8.1$.

\section{2'-O-Ac-3-OH-6-O-methylerythromycin A 9-O-(2-chlorobenzyl)oxime (5)}

A solution of $4(5.0 \mathrm{~g}, 6.9 \mathrm{mmol})$ in $\mathrm{CH}_{2} \mathrm{Cl}_{2}(50 \mathrm{ml})$ was treated with acetic anhydride $(1.7 \mathrm{ml}, 17.9 \mathrm{mmol})$ at room temperature for $1 \mathrm{~h}$. The reaction mixture was washed with saturated $\mathrm{NaHCO}_{3}$ and brine. The organic layer was dried over $\mathrm{MgSO}_{4}$ and evaporated to yield 5 (5.0 g, 94.5\%). 


\section{2'-O-Ac-3-OH-6-O-methylerythromycin A}

\section{9-O-(2-chlorobenzyl)oxime 11,12-carbonate (6)}

To an ice-cold solution of $5(4.8 \mathrm{~g}, 6.3 \mathrm{mmol})$ in $\mathrm{CH}_{2} \mathrm{Cl}_{2}(120 \mathrm{ml})$ was added pyridine $(5.0 \mathrm{ml}, 61.9 \mathrm{mmol})$ and dropped by a solution of Bis(trichloromethyl)carbonate $(3.73 \mathrm{~g}, 12.5 \mathrm{mmol})$ in $\mathrm{CH}_{2} \mathrm{Cl}_{2}(60 \mathrm{ml})$ over $1 \mathrm{~h}$. The reaction mixture was stirred at room temperature for $18 \mathrm{~h}$ and then cooled to $0^{\circ} \mathrm{C}$. To the reaction mixture was added dropwise water $(100 \mathrm{ml})$. The organic layer was washed with saturated $\mathrm{NaHCO}_{3}$ and water, and then dried over $\mathrm{MgSO}_{4}$ and evaporated to yield 6 ( $4.9 \mathrm{~g}, 98.7 \%)$.

\section{2'-O-Ac-3-O-allyl-6-O-methylerythromycin A \\ 9-O-(2-chlorobenzyl)oxime 11,12-carbonate (7)}

To an ice-cold solution of $6(2.0 \mathrm{~g}, 2.5 \mathrm{mmol})$ in $20 \mathrm{ml}$ of DMSO and $20 \mathrm{ml}$ THF was added allyl bromide $(0.42 \mathrm{ml}, 5.0 \mathrm{mmol})$ and $\mathrm{KOtBu}(0.56 \mathrm{~g}$, $5.0 \mathrm{mmol}$ ). The reaction mixture was stirred for $30 \mathrm{~min}$, and then poured with water and extracted with ethyl acetate. The organic phase was washed with water and brine. The organic layer was dried over $\mathrm{MgSO}_{4}$ and concentrated to yield $7(2.0 \mathrm{~g}, 95.2 \%)$. HRMS (ESI) $\left(\mathrm{M}+\mathrm{H}^{+}\right) \mathrm{m} / \mathrm{z}$ 837.42967, calcd for $\mathrm{C}_{43} \mathrm{H}_{66} \mathrm{ClN}_{2} \mathrm{O}_{12}$ 837.42988. ${ }^{1} \mathrm{H}$ NMR $\left(400 \mathrm{MHz}, \mathrm{CDCl}_{3}\right) \delta: 0.82-0.91(\mathrm{~m}$, $\left.9 \mathrm{H}, 15-\mathrm{CH}_{3}, 8-\mathrm{CH}_{3}, 4-\mathrm{CH}_{3}\right), 1.18-1.30\left(\mathrm{~m}, 13 \mathrm{H}, 10-\mathrm{CH}_{3}, 5^{\prime}-\mathrm{CH}_{3}, 2-\mathrm{CH}_{3}, 6-\right.$ $\left.\mathrm{CH}_{3}, \mathrm{H}-7\right), 1.46\left(\mathrm{~s}, 3 \mathrm{H}, 12-\mathrm{CH}_{3}\right), 1.51-1.56(\mathrm{~m}, 1 \mathrm{H}, \mathrm{H}-14 \mathrm{ax}), 1.67$ (dd, $\left.J=2.7 \mathrm{~Hz}, 1 \mathrm{H}, \mathrm{H}-4^{\prime}\right), 1.83-1.88(\mathrm{~m}, 2 \mathrm{H}, \mathrm{H}-4, \mathrm{H}-14 \mathrm{eq}), 2.02\left(\mathrm{~s}, 3 \mathrm{H}, 2^{\prime}-\mathrm{COCH}_{3}\right)$ $2.24\left(\mathrm{~s}, 6 \mathrm{H},-\mathrm{N}\left(\mathrm{CH}_{3}\right)_{2}\right), 2.44(\mathrm{q}, 1 \mathrm{H}, \mathrm{H}-10), 2.62-2.64\left(\mathrm{~m}, 1 \mathrm{H}, \mathrm{H}-3^{\prime}\right), 2.68(\mathrm{~s}$, $\left.3 \mathrm{H}, 6-\mathrm{OCH}_{3}\right), 2.78-2.82(\mathrm{~m}, 1 \mathrm{H}, \mathrm{H}-2), 3.19$ (d, $\left.J=10.5 \mathrm{~Hz}, 1 \mathrm{H}, \mathrm{H}-3\right), 3.39-3.45$ $\left(\mathrm{m}, 1 \mathrm{H}, \mathrm{H}-5^{\prime}\right), 3.62(\mathrm{~d}, J=3.2 \mathrm{~Hz}, 1 \mathrm{H}, \mathrm{H}-5), 3.72(\mathrm{~m}, 1 \mathrm{H}, \mathrm{H}-8), 4.05$ and 4.27 (dd, $J=5.1$ and $\left.13.2 \mathrm{~Hz}, 2 \mathrm{H},-\mathrm{CH}_{2}-\mathrm{CH}=\mathrm{CH}_{2}\right), 4.41\left(\mathrm{~d}, J=7.5 \mathrm{~Hz}, 1 \mathrm{H}, \mathrm{H}-\mathrm{I}^{\prime}\right)$, $4.70\left(\mathrm{dd}, J=7.5\right.$ and $\left.10.5 \mathrm{~Hz}, 1 \mathrm{H}, \mathrm{H}-2^{\prime}\right), 4.78(\mathrm{~s}, 1 \mathrm{H}, \mathrm{H}-11), 5.08-5.24(\mathrm{~m}, 3 \mathrm{H}$, $\mathrm{H}-13$, benzyl- $\mathrm{CH}_{2}$ ), $5.34\left(\mathrm{dd}, J=1.6\right.$ and $\left.17.2 \mathrm{~Hz}, 1 \mathrm{H},-\mathrm{CH}=\mathrm{CH}_{2}\right), 5.83-5.93$ $\left(\mathrm{m}, 1 \mathrm{H},-\mathrm{CH}=\mathrm{CH}_{2}\right),[7.48-7.50(\mathrm{~m}, 1 \mathrm{H}), 7.30(\mathrm{~d}, J=1.2$ and $7.6 \mathrm{~Hz}, 1 \mathrm{H}), 7.15-$ $7.26(\mathrm{~m}, 2 \mathrm{H})$, benzyl ring $.{ }^{13} \mathrm{C} \mathrm{NMR}\left(100 \mathrm{MHz}, \mathrm{CDCl}_{3}\right) \delta: 175.2(\mathrm{C}-1)$, 169.7(C-9), 165.3 $\left(-\mathrm{COCH}_{3}\right)$, 154.6(carbonate), 136.1, 134.4, 133.4, 130.8, $128.9,128.6,126.4,116.4,99.2,84.9,84.2,82.8,79.0,78.4,75.1,74.4,72.6$, 71.4, 68.5, 49.4, 44.7, 40.6(- $\left.\left(\mathrm{CH}_{3}\right)_{2}\right), 36.9,36.6,32.8,31.0,25.8,22.1,21.1$, $20.9,19.3,18.8,15.5,15.2,12.8,10.1,8.5$.

\section{3-O-[3-(3'-Quinolyl)-Z-1-propenyl]-6-O-methylerythromycin A 9-O-(2-chlorobenzyl)oxime 11, 12-carbonate $(9 \mathrm{e})$}

To a solution of $7(1.0 \mathrm{~g}, 1.2 \mathrm{mmol})$, palladium(II) acetate $(53.6 \mathrm{mg}, 0.24 \mathrm{mmol})$ and tri $(o$-tolyl $)$ phosphine $(145.36 \mathrm{mg}, 0.48 \mathrm{mmol})$, in acetonitrile $(8 \mathrm{ml})$ were added 3-bromoquinoline $(0.324 \mathrm{ml}, 2.4 \mathrm{mmol})$ and triethylamine $(0.33 \mathrm{ml}$, $2.4 \mathrm{mmol})$. The reaction mixture was flushed with nitrogen and sealed in a pressure tube. The reaction mixture was stirred at $60^{\circ} \mathrm{C}$ for $1 \mathrm{~h}$ and thereafter at $90^{\circ} \mathrm{C}$ for $24 \mathrm{~h}$. The reaction mixture was extracted with ethyl acetate, washed with water and brine and the organic phase was concentrated. The crude mixture was purified by column chromatography on silica gel (15:0.4:0.1 $\left.\mathrm{CH}_{2} \mathrm{Cl}_{2} / \mathrm{C}_{2} \mathrm{H}_{5} \mathrm{OH} / \mathrm{NH}_{3} \cdot \mathrm{H}_{2} \mathrm{O}\right)$ to yield $8 \mathrm{e}(216 \mathrm{mg}, 19.4 \%)$.

The product obtained above $(216 \mathrm{mg}, 0.22 \mathrm{mmol})$ was heated to reflux in $\mathrm{MeOH}(20 \mathrm{ml})$ for $3 \mathrm{~h}$. After the solvent was evaporated, the crude product was purified by column chromatography (silica gel, 5:5:0.2 petroleum ether/ acetone/triethylamine) to yield 9e (100 mg, 48.4\%): MS(MALDI-TOF) $\left(\mathrm{M}+\mathrm{Na}^{+}\right) \mathrm{m} / z$ 944.7. HRMS (ESI) $\left(\mathrm{M}+\mathrm{H}^{+}\right) \mathrm{m} / \mathrm{z}$ 922.46049, calcd for $\mathrm{C}_{50} \mathrm{H}_{69} \mathrm{ClN}_{3} \mathrm{O}_{11}$ 922.46151. ${ }^{1} \mathrm{H}$ NMR $\left(400 \mathrm{MHz}, \mathrm{CDCl}_{3}\right) \delta: 0.85$ (t, 3H, 15$\left.\mathrm{CH}_{3}\right), 0.93\left(\mathrm{~d}, J=6.9 \mathrm{~Hz}, 3 \mathrm{H}, 8-\mathrm{CH}_{3}\right), 1.12-1.20\left(\mathrm{~m}, 9 \mathrm{H}, 4-\mathrm{CH}_{3}, 2-\mathrm{CH}_{3}, 5^{\prime}-\right.$ $\left.\mathrm{CH}_{3}\right), 1.22\left(\mathrm{~d}, J=6.8 \mathrm{~Hz}, 3 \mathrm{H}, 10-\mathrm{CH}_{3}\right), 1.28\left(\mathrm{~s}, 3 \mathrm{H}, 6-\mathrm{CH}_{3}\right), 1.32-1.35(\mathrm{~m}, 1 \mathrm{H}$, H-7), 1.42-1.60 (m, 6H, H-7, 12- $\left.\mathrm{CH}_{3}, \mathrm{H}-14 \mathrm{ax}, \mathrm{H}-4^{\prime}\right), 1.88-1.99$ (m, 2H, H-4, H-14eq), 2.14-2.17 (m, 7H, -N( $\left.\left.\mathrm{CH}_{3}\right)_{2}, \mathrm{H}-3^{\prime}\right), 2.47$ (q, 1H, H-10), 2.71 (s, 3H, 6- $\left.\mathrm{OCH}_{3}\right), 2.89-2.96(\mathrm{~m}, 1 \mathrm{H}, \mathrm{H}-2), 3.12\left(\mathrm{dd}, J=7.2\right.$ and $\left.10.1 \mathrm{~Hz}, 1 \mathrm{H}, \mathrm{H}-2^{\prime}\right)$ $3.33-3.36\left(\mathrm{~m}, 2 \mathrm{H}, \mathrm{H}-5^{\prime}, 3-\mathrm{O}-\mathrm{CH}=\mathrm{CH}-\mathrm{CH}_{2}-\mathrm{Ar}\right), 3.49-3.55(\mathrm{~m}, 2 \mathrm{H},-3-\mathrm{O}-$ $\left.\mathrm{CH}=\mathrm{CH}-\mathrm{CH}_{2}-\mathrm{Ar}, \mathrm{H}-3\right), 3.66(\mathrm{~d}, J=2.7 \mathrm{~Hz}, 1 \mathrm{H}, \mathrm{H}-5), 3.67-3.75\left(\mathrm{~m}, 2 \mathrm{H}, 2^{\prime}-\right.$ $\mathrm{OH}, \mathrm{H}-8), 4.21\left(\mathrm{~d}, J=7.3 \mathrm{~Hz}, 1 \mathrm{H}, \mathrm{H}-\mathrm{l}^{\prime}\right), 4.62\left(\mathrm{q}, 1 \mathrm{H}, 3-\mathrm{O}-\mathrm{CH}=\mathrm{CH}-\mathrm{CH}_{2}-\mathrm{Ar}\right)$, 4.83 (s, $1 \mathrm{H}, \mathrm{H}-11)$, 5.09-5.25 (m, 3H, $\left.-\mathrm{CH}_{2}-\mathrm{PhCl}, \mathrm{H}-13\right), 6.25$ (d, $J=6.5 \mathrm{~Hz}$, $\left.1 \mathrm{H}, 3-\mathrm{O}-\mathrm{CH}=\mathrm{CH}-\mathrm{CH}_{2}-\mathrm{Ar}\right),[7.19-7.25(\mathrm{~m}, 2 \mathrm{H}), 7.32(\mathrm{dd}, J=1.3$ and $5.2 \mathrm{~Hz}$, $1 \mathrm{H}), 7.52-7.54(\mathrm{~m}, 1 \mathrm{H})$, benzyl ring], [7.52-7.54 $(\mathrm{m}, 1 \mathrm{H}), 7.64(\mathrm{~m}, 1 \mathrm{H}), 7.75(\mathrm{~d}$, $J=8.0 \mathrm{~Hz}, 1 \mathrm{H}), 7.94(\mathrm{~s}, 1 \mathrm{H}), 8.05(\mathrm{~d}, J=8.3 \mathrm{~Hz}, 1 \mathrm{H}), 8.82(\mathrm{~d}, J=2.1 \mathrm{~Hz}, 1 \mathrm{H})$, quinolyl].
3-O-[3-(4'-Isoquinolyl)-Z-1-propenyl]-6-O-methylerythromycin A 9-O-(2-chlorobenzyl)oxime 11,12-carbonate (9f)

By a similar procedure to $\mathbf{9 e}, \mathbf{8 f}$ was prepared as a white solid in $17.1 \%$ yield according to the general procedure for the preparation of $\mathbf{8 e}$, and then deacetylated to give 9f $(38.6 \%)$. HRMS (ESI) $\left(\mathrm{M}+\mathrm{H}^{+}\right) \mathrm{m} / \mathrm{z}$ 922.46301, calcd for $\mathrm{C}_{50} \mathrm{H}_{69} \mathrm{ClN}_{3} \mathrm{O}_{11}$ 922.46151. ${ }^{1} \mathrm{H}$ NMR $\left(400 \mathrm{MHz}, \mathrm{CDCl}_{3}\right) \delta$ : $0.85(\mathrm{t}, 3 \mathrm{H}$, $\left.15-\mathrm{CH}_{3}\right), 0.93\left(\mathrm{~d}, J=6.9 \mathrm{~Hz}, 3 \mathrm{H}, 8-\mathrm{CH}_{3}\right), 1.12-1.22\left(\mathrm{~m}, 9 \mathrm{H}, 4-\mathrm{CH}_{3}, 2-\mathrm{CH}_{3}\right.$, $\left.5^{\prime}-\mathrm{CH}_{3}\right), 1.23\left(\mathrm{~d}, J=6.8 \mathrm{~Hz}, 3 \mathrm{H}, 10-\mathrm{CH}_{3}\right), 1.29\left(\mathrm{~s}, 3 \mathrm{H}, 6-\mathrm{CH}_{3}\right), 1.35(\mathrm{~d}, 1 \mathrm{H}, \mathrm{H}-7)$, 1.45 (d, 1H, H-7), 1.51 (s, 3H, 12- $\left.\mathrm{CH}_{3}\right), 1.56$ (m, 2H, H-4', H-14ax), 1.87-1.93 (m, 1H, H-14eq), 1.99-2.03 (m, 1H, H-4), 2.23 (s, 6H, $\left.-\mathrm{N}\left(\mathrm{CH}_{3}\right)_{2}\right), 2.25-2.26$ $\left(\mathrm{m}, 1 \mathrm{H}, \mathrm{H}-3^{\prime}\right), 2.48(\mathrm{q}, 1 \mathrm{H}, \mathrm{H}-10), 2.71\left(\mathrm{~s}, 3 \mathrm{H}, 6-\mathrm{OCH}_{3}\right), 2.95-2.99(\mathrm{~m}, 1 \mathrm{H}, \mathrm{H}-$ 2), $3.14\left(\mathrm{dd}, J=7.3\right.$ and $\left.10.0 \mathrm{~Hz}, 1 \mathrm{H}, \mathrm{H}-2^{\prime}\right), 3.31-3.35\left(\mathrm{~m}, 2 \mathrm{H}, \mathrm{H}-5^{\prime}, 2^{\prime}-\mathrm{OH}\right)$, $3.55(\mathrm{~d}, J=10.5 \mathrm{~Hz}, 1 \mathrm{H}, \mathrm{H}-3), 3.66-3.72\left(\mathrm{~m}, 2 \mathrm{H}, \mathrm{H}-5,3-\mathrm{O}-\mathrm{CH}=\mathrm{CH}-\mathrm{CH}_{2}-\mathrm{Ar}\right)$, $3.77(\mathrm{~m}, 1 \mathrm{H}, \mathrm{H}-8), 3.94\left(\mathrm{dd}, J=7.6\right.$ and $15.6 \mathrm{~Hz}, 1 \mathrm{H}, 3-\mathrm{O}-\mathrm{CH}=\mathrm{CH}-\mathrm{CH}_{2}-\mathrm{Ar}$ ), $4.26\left(\mathrm{~d}, J=7.2 \mathrm{~Hz}, 1 \mathrm{H}, \mathrm{H}-\mathrm{1}^{\prime}\right), 4.60\left(\mathrm{q}, 1 \mathrm{H}, 3-\mathrm{O}-\mathrm{CH}=\mathrm{CH}-\mathrm{CH}_{2}-\mathrm{Ar}\right), 4.85(\mathrm{~s}, 1 \mathrm{H}$, $\mathrm{H}-11), 5.10-5.16\left(\mathrm{~m}, 2 \mathrm{H},-\mathrm{CH}_{2}-\mathrm{PhCl}, \mathrm{H}-13\right), 5.24\left(\mathrm{~d}, J=12.9 \mathrm{~Hz}, 1 \mathrm{H},-\mathrm{CH}_{2}-\right.$ $\mathrm{PhCl}), 6.19\left(\mathrm{~d}, J=6.1 \mathrm{~Hz}, 1 \mathrm{H}, 3-\mathrm{O}-\mathrm{CH}=\mathrm{CH}-\mathrm{CH}_{2}-\mathrm{Ar}\right),[7.12-7.25(\mathrm{~m}, 2 \mathrm{H})$, $7.32(\mathrm{dd}, J=1.1$ and $7.9 \mathrm{~Hz}, 1 \mathrm{H}), 7.53(\mathrm{dd}, J=1.3$ and $7.5 \mathrm{~Hz}, 1 \mathrm{H})$, benzyl ring], [7.63 (t, 1H), $7.74(\mathrm{~m}, 1 \mathrm{H}), 7.98(\mathrm{~d}, J=8.1 \mathrm{~Hz}, 1 \mathrm{H}), 8.07(\mathrm{~d}, J=8.4 \mathrm{~Hz}, 1 \mathrm{H})$, $8.42(\mathrm{~s}, 1 \mathrm{H}), 9.13(\mathrm{~s}, 1 \mathrm{H})$, isoquinolyl]. ${ }^{13} \mathrm{C} \mathrm{NMR}\left(100 \mathrm{MHz}, \mathrm{CDCl}_{3}\right) \delta: 174.4$ (C-1), 165.1 (C-9), 154.6 (carbonate), 151.4, 148.1, 142.3, 136.0, 134.5, 133.5, 131.0, 130.3, 130.2, 128.9, 128.7, 128.3, 128.1, 126.9, 126.4, 123.1, 102.1, 101.6, $89.5,84.9,82.8,79.7,78.4,77.1,75.4,72.6,70.4,69.2,65.6,49.3,44.5,40.2$ $\left(-\mathrm{N}\left(\mathrm{CH}_{3}\right)_{2}\right), 37.1,36.5,32.8,28.4,25.9,25.2,22.1,21.1,19.3,18.8,15.6,15.4$, $12.8,10.0,8.9$.

\section{3-O-[3-(5'-Isoquinolyl)-Z-1-propenyl]-6-O-methylerythromycin A} 9-O-(2-chlorobenzyl)oxime 11,12-carbonate (9g)

By a similar prodedure to $\mathbf{9 e}, \mathbf{8} \mathbf{g}$ was prepared as a white solid in $18.0 \%$ yield according to the general procedure for the preparation of $\mathbf{8 e}$, and then deacetylated to give $\mathbf{9 g}(52.3 \%)$. HRMS (ESI) $\left(\mathrm{M}+\mathrm{H}^{+}\right) \mathrm{m} / \mathrm{z}$ 922.46176, calcd for $\mathrm{C}_{50} \mathrm{H}_{69} \mathrm{ClN}_{3} \mathrm{O}_{11}$ 922.46151. ${ }^{1} \mathrm{H}$ NMR $\left(600 \mathrm{MHz}, \mathrm{CDCl}_{3}\right) \delta: 0.86(\mathrm{t}, 3 \mathrm{H}$, $\left.15-\mathrm{CH}_{3}\right), 0.94\left(\mathrm{~d}, J=7.6 \mathrm{~Hz}, 3 \mathrm{H}, 8-\mathrm{CH}_{3}\right), 1.13\left(\mathrm{~d}, J=6.9 \mathrm{~Hz}, 3 \mathrm{H}, 4-\mathrm{CH}_{3}\right), 1.16-$ $1.18\left(\mathrm{~m}, 9 \mathrm{H}, 2-\mathrm{CH}_{3}, 4-\mathrm{CH}_{3}, 5^{\prime}-\mathrm{CH}_{3}\right), 1.21-1.25\left(\mathrm{~m}, 3 \mathrm{H}, 10-\mathrm{CH}_{3}\right), 1.31(\mathrm{~s}, 3 \mathrm{H}$, 6- $\left.\mathrm{CH}_{3}\right), 1.35-1.31(\mathrm{~m}, 1 \mathrm{H}, \mathrm{H}-7), 1.43-1.48(\mathrm{~m}, 1 \mathrm{H}, \mathrm{H}-7), 1.51(\mathrm{~s}, 3 \mathrm{H}, 12-$ $\mathrm{CH}_{3}$ ), 1.54-1.61 (m, 2H, H-14ax, H-4'), 1.89-1.93 (m, 1H, H-14eq), 1.97-2.02 $(\mathrm{m}, 1 \mathrm{H}, \mathrm{H}-4), 2.18\left(\mathrm{~m}, 7 \mathrm{H}, \mathrm{H}-3^{\prime},-\mathrm{N}\left(\mathrm{CH}_{3}\right)_{2}\right), 2.48(\mathrm{q}, 1 \mathrm{H}, \mathrm{H}-10), 2.71(\mathrm{~s}, 3 \mathrm{H}$, 6- $\left.\mathrm{OCH}_{3}\right), 2.92-2.95(\mathrm{~m}, 1 \mathrm{H}, \mathrm{H}-2), 3.14\left(\mathrm{dd}, J=6.9\right.$ and $\left.10.3 \mathrm{~Hz}, 1 \mathrm{H}, \mathrm{H}-2^{\prime}\right)$, 3.28-3.32 (m, 2H, 2'-OH, H-5'), 3.53 (d, $J=10.9 \mathrm{~Hz}, 1 \mathrm{H}, \mathrm{H}-3), 3.67-3.71(\mathrm{~m}$, $\left.2 \mathrm{H}, \mathrm{H}-5,3-\mathrm{O}-\mathrm{CH}=\mathrm{CH}-\mathrm{CH}_{2}-\mathrm{Ar}\right), 3.76-3.78(\mathrm{~m}, 1 \mathrm{H}, \mathrm{H}-8), 3.98(\mathrm{dd}, J=7.5$ and $\left.15.8 \mathrm{~Hz}, 1 \mathrm{H}, 3-\mathrm{O}-\mathrm{CH}=\mathrm{CH}-\mathrm{CH}_{2}-\mathrm{Ar}\right), 4.25\left(\mathrm{~d}, J=7.5 \mathrm{~Hz}, 1 \mathrm{H}, \mathrm{H}-\mathrm{l}^{\prime}\right), 4.57-$ $4.60\left(\mathrm{~m}, 1 \mathrm{H}, 3-\mathrm{O}-\mathrm{CH}=\mathrm{CH}-\mathrm{CH}_{2}-\mathrm{Ar}\right), 4.84(\mathrm{~s}, 1 \mathrm{H}, \mathrm{H}-11), 5.11-5.29(\mathrm{~m}, 2 \mathrm{H}$, $\mathrm{H}-13,-\mathrm{CH}_{2}-\mathrm{PhCl}$ ), 6.20 (d, $J=6.2 \mathrm{~Hz}, 1 \mathrm{H}, 3-\mathrm{O}-\mathrm{CH}=\mathrm{CH}-\mathrm{CH}_{2}-\mathrm{Ar}$ ), [7.19$7.25(\mathrm{~m}, 2 \mathrm{H}), 7.32(\mathrm{~d}, J=7.5 \mathrm{~Hz}, 1 \mathrm{H}), 7.51-7.54(\mathrm{~m}, 1 \mathrm{H})$, benzyl ring], [7.51$7.54(\mathrm{~m}, 1 \mathrm{H}), 7.59(\mathrm{~d}, J=6.8 \mathrm{~Hz}, 1 \mathrm{H}), 7.84(\mathrm{~d}, J=8.2 \mathrm{~Hz}, 1 \mathrm{H}), 7.89(\mathrm{~d}, J=6.2 \mathrm{~Hz}$ $1 \mathrm{H}), 8.56(\mathrm{~d}, J=5.5 \mathrm{~Hz}, 1 \mathrm{H}), 9.24(\mathrm{~s}, 1 \mathrm{H})$, isoquinolyl]

\section{3-O-[3-(5'-Indolyl)-E-2-propenyl]-6-O-methylerythromycin A 9-O-(2-chlorobenzyl)oxime 11,12-carbonate $(9 \mathrm{~h})$}

By a similar prodedure to $\mathbf{9 e}, \mathbf{8} \mathbf{h}$ was prepared as a white solid in $21.0 \%$ yield according to the general procedure for the preparation of $\mathbf{8 e}$, and then deacetylated to give $9 \mathbf{h}(13.9 \%)$. HRMS (ESI) $\left(\mathrm{M}+\mathrm{H}^{+}\right) \mathrm{m} / \mathrm{z}$ 910.46418, calcd for $\mathrm{C}_{49} \mathrm{H}_{69} \mathrm{ClN}_{3} \mathrm{O}_{11}$ 910.46151. ${ }^{1} \mathrm{H}$ NMR $\left(400 \mathrm{MHz}, \mathrm{CDCl}_{3}\right) \delta: 0.87(\mathrm{t}, 3 \mathrm{H}, 15-$ $\left.\mathrm{CH}_{3}\right), 0.91\left(\mathrm{~d}, J=6.9 \mathrm{~Hz}, 3 \mathrm{H}, 8-\mathrm{CH}_{3}\right), 1.09\left(\mathrm{~d}, J=7.5 \mathrm{~Hz}, 3 \mathrm{H}, 4-\mathrm{CH}_{3}\right), 1.22-1.19$ $\left(\mathrm{m}, 6 \mathrm{H}, 5^{\prime}-\mathrm{CH}_{3}, 2-\mathrm{CH}_{3}\right), 1.25-1.34\left(\mathrm{~m}, 6 \mathrm{H}, 10-\mathrm{CH}_{3}, 6-\mathrm{CH}_{3}\right), 1.42(\mathrm{~m}, 1 \mathrm{H}, \mathrm{H}-$ 7), $1.49\left(\mathrm{~s}, 3 \mathrm{H}, 12-\mathrm{CH}_{3}\right), 1.56\left(\mathrm{~m}, 3 \mathrm{H}, \mathrm{H}-7, \mathrm{H}-14 \mathrm{ax}, \mathrm{H}-4^{\prime}\right), 1.92-1.87(\mathrm{~m}, 2 \mathrm{H}$, $\mathrm{H}-14 \mathrm{eq}, \mathrm{H}-4), 2.10\left(\mathrm{~s}, 6 \mathrm{H},-\mathrm{N}\left(\mathrm{CH}_{3}\right)_{2}\right), 2.37$ (br, $\left.1 \mathrm{H}, \mathrm{H}-3^{\prime}\right), 2.47(\mathrm{q}, 1 \mathrm{H}, \mathrm{H}-10)$, $2.63\left(\mathrm{~s}, 3 \mathrm{H}, 6-\mathrm{OCH}_{3}\right), 2.92-2.87(\mathrm{~m}, 1 \mathrm{H}, \mathrm{H}-2), 3.16\left(\mathrm{~m}, 1 \mathrm{H}, \mathrm{H}-2^{\prime}\right), 3.28(\mathrm{~s}, 1 \mathrm{H}$, $\left.2^{\prime}-\mathrm{OH}\right), 3.34$ (d, $\left.J=7.8 \mathrm{~Hz}, 1 \mathrm{H}, \mathrm{H}-3\right), 3.46\left(\mathrm{~m}, 1 \mathrm{H}, \mathrm{H}-5^{\prime}\right), 3.71$ (m, 2H, H-8, H-5), $4.23\left(\mathrm{dd}, J=5.3\right.$ and $\left.13.2 \mathrm{~Hz}, 1 \mathrm{H}, 3-\mathrm{O}-\mathrm{CH}_{2}-\mathrm{CH}=\mathrm{CH}-\mathrm{Ar}\right), 4.48(\mathrm{~d}, J=5.4 \mathrm{~Hz}, 1 \mathrm{H}$, $\left.\mathrm{H}-1^{\prime}\right), 4.53$ (dd, $J=9.3 \mathrm{~Hz}, 1 \mathrm{H}, 3-\mathrm{O}-\mathrm{CH}_{2}-\mathrm{CH}=\mathrm{CH}-\mathrm{Ar}$ ), $4.82(\mathrm{~s}, 1 \mathrm{H}, \mathrm{H}-11$ ), $5.08-5.15\left(\mathrm{~m}, 2 \mathrm{H},-\mathrm{CH}_{2}-\mathrm{PhCl}, \mathrm{H}-13\right), 5.23\left(\mathrm{~d}, J=12.9 \mathrm{~Hz}, 1 \mathrm{H},-\mathrm{CH}_{2}-\mathrm{PhCl}\right), 6.29$ $\left(\mathrm{dt}, J=15.6 \mathrm{~Hz}, 1 \mathrm{H}, 3-\mathrm{O}-\mathrm{CH}_{2}-\mathrm{CH}=\mathrm{CH}-\mathrm{Ar}\right), 6.78\left(\mathrm{~d}, J=15.9 \mathrm{~Hz}, 1 \mathrm{H}, 3-\mathrm{O}-\mathrm{CH}_{2}-\right.$ $\mathrm{CH}=\mathrm{CH}-\mathrm{Ar}),[6.52(\mathrm{~m}, 1 \mathrm{H}), 7.12(\mathrm{~m}, 1 \mathrm{H}), 7.20-7.31(\mathrm{~m}, 4 \mathrm{H}), 7.34-7.36(\mathrm{~m}$, $1 \mathrm{H}), 7.50-7.53(\mathrm{~m}, 1 \mathrm{H}), 7.62(\mathrm{~s}, 1 \mathrm{H}), 8.19(\mathrm{~s}, 1 \mathrm{H})$, benzyl ring, indolyl]. ${ }^{13} \mathrm{C}$ NMR $\left(100 \mathrm{MHz}, \mathrm{CDCl}_{3}\right) \delta: 175.4$ (C-1), 165.3(C-9), 154.8 (carbonate), 136.1, 
$135.5,133.5,133.2,131.0,128.9,128.6,128.5,128.1,126.4,124.8,122.6,120.4$, $119.2,111.1,102.7,101.7,85.0,83.8,82.9,80.2,78.3,77.1,75.0,74.2,72.6,70.5$, 69.1, 65.1, 49.3, 44.9, $39.9\left(-\mathrm{N}\left(\mathrm{CH}_{3}\right)_{2}\right), 37.2,36.9,32.8,28.6,25.9,22.1,21.3,19.4$, $18.8,15.6,15.5,12.8,10.1,8.8$

\section{3-O-[3-(5'-Pyrimidyl)-Z-1-propenyl]-6-O-methylerythromycin A 9-O-(2-chlorobenzyl)oxime 11,12-carbonate (9d)}

By a similar prodedure to $\mathbf{9 e ,} \mathbf{8 d}$ was prepared as a white solid in $9.4 \%$ yield according to the general procedure for the preparation of $8 \mathbf{e}$, except for the addition of 3eq 5-bromopyrimidine, and then deacetylated to give $9 \mathbf{d}(32.3 \%)$. HRMS (ESI) $\left(\mathrm{M}+\mathrm{H}^{+}\right) \mathrm{m} / z$ 873.44271, calcd for $\mathrm{C}_{45} \mathrm{H}_{66} \mathrm{ClN}_{4} \mathrm{O}_{11} 873.44111$. ${ }^{1} \mathrm{H}$ NMR $\left(400 \mathrm{MHz}, \mathrm{CDCl}_{3}\right), \delta: 0.83\left(\mathrm{t}, 3 \mathrm{H}, 15-\mathrm{CH}_{3}\right), 0.91\left(\mathrm{~d}, 3 \mathrm{H}, 8-\mathrm{CH}_{3}\right)$, 1.04-1.11 (m, 6H, 4- $\left.\mathrm{CH}_{3}, 2-\mathrm{CH}_{3}\right), 1.17-1.24\left(\mathrm{~m}, 7 \mathrm{H}, 10-\mathrm{CH}_{3}, 5^{\prime}-\mathrm{CH}_{3}, \mathrm{H}-4^{\prime} \mathrm{ax}\right)$, $1.27\left(\mathrm{~s}, 3 \mathrm{H}, 6-\mathrm{CH}_{3}\right), 1.42-1.28(\mathrm{~m}, 2 \mathrm{H}, \mathrm{H}-7), 1.48\left(\mathrm{~s}, 3 \mathrm{H}, 12-\mathrm{CH}_{3}\right), 1.61$ (m, 2H, H-14ax, H-4'eq), 1.86-1.96 (m, 2H, H-14eq, H-4), 2.25 (s, 6H, -N( $\left.\left.\mathrm{CH}_{3}\right)_{2}\right)$, 2.31 (m, 1H, H-3'), 2.46 (q, 1H, H-10), 2.69 (s, 3H, 6- $\left.-\mathrm{OCH}_{3}\right), 2.86-2.91(\mathrm{~m}, 1 \mathrm{H}$, $\mathrm{H}-2), 3.14$ (dd, $J=7.3$ and $\left.15.9 \mathrm{~Hz}, 1 \mathrm{H}, \mathrm{H}-2^{\prime}\right), 3.25-3.35\left(\mathrm{~m}, 2 \mathrm{H}, \mathrm{H}-5^{\prime}, 3-\mathrm{O}-\right.$ $\left.\mathrm{CH}=\mathrm{CH}-\mathrm{CH}_{2}-\mathrm{Ar}\right), 3.48-3.52\left(\mathrm{~m}, 2 \mathrm{H}, 3-\mathrm{O}-\mathrm{CH}=\mathrm{CH}-\mathrm{CH}_{2}-\mathrm{Ar}, \mathrm{H}-3\right), 3.61$ (d, $J=2.4 \mathrm{~Hz}, 1 \mathrm{H}, \mathrm{H}-5), 3.75(\mathrm{~m}, 1 \mathrm{H}, \mathrm{H}-8), 4.16\left(\mathrm{~d}, J=7.2 \mathrm{~Hz}, 1 \mathrm{H}, \mathrm{H}-1^{\prime}\right)$, $4.52\left(\mathrm{q}, 1 \mathrm{H}, 3-\mathrm{O}-\mathrm{CH}=\mathrm{CH}-\mathrm{CH}_{2}-\mathrm{Ar}\right), 4.81(\mathrm{~s}, 1 \mathrm{H}, \mathrm{H}-11), 4.78-4.81(\mathrm{~m}, 2 \mathrm{H},-$ $\left.\mathrm{CH}_{2}-\mathrm{PhCl}, \mathrm{H}-13\right), 5.22\left(\mathrm{~d}, J=12.9 \mathrm{~Hz}, 1 \mathrm{H},-\mathrm{CH}_{2}-\mathrm{PhCl}\right), 6.22(\mathrm{~d}, J=6.0 \mathrm{~Hz}, 1 \mathrm{H}$, $\left.3-\mathrm{O}-\mathrm{CH}=\mathrm{CH}-\mathrm{CH}_{2}-\mathrm{Ar}\right),[7.16-7.23(\mathrm{~m}, 2 \mathrm{H}), 7.29-7.31(\mathrm{~m}, 1 \mathrm{H}), 7.50-7.52$ $(\mathrm{m}, 1 \mathrm{H})$, benzyl ring], [8.60 (s, 2H), $9.04(\mathrm{~s}, 1 \mathrm{H})$, pyrimidyl]. ${ }^{13} \mathrm{C}$ NMR $\left(100 \mathrm{MHz}, \mathrm{CDCl}_{3}\right) \delta: 174.2$ (C-1), 165.1 (C-9), 156.6, 154.6 (carbonate), 149.2, $136.0,134.4,133.5,130.9,128.9,128.7,126.4,101.8,100.5,89.8,84.8,82.8$, 80.1, 78.4, 77.1, 75.5, 72.6, 70.2, 69.2, 65.8, 49.3, 44.4, 40.2 (-N( $\left.\left.\mathrm{CH}_{3}\right)_{2}\right), 37.1$, $36.4,32.8,28.4,25.8,25.3,22.1,21.1,19.3,18.7,15.6,15.4,12.8,10.0,8.8$.

\section{3-O-[3-(1'-Phenyl)-Z-1-propenyl]-6-O-methylerythromycin A 9-O-(2-chlorobenzyl)oxime 11,12 -carbonate (9b)}

By a similar prodedure to $\mathbf{9 e}, \mathbf{8 b}$ was prepared as a white solid in $51.2 \%$ yield according to the general procedure for the preparation of $\mathbf{8 e}$, and then deacetylated to give $\mathbf{9 b}(16.2 \%)$. HRMS (ESI) $\left(\mathrm{M}+\mathrm{H}^{+}\right) \mathrm{m} / \mathrm{z} 871.45056$, calcd for $\mathrm{C}_{47} \mathrm{H}_{68} \mathrm{ClN}_{2} \mathrm{O}_{11}$ 871.45062. ${ }^{1} \mathrm{H}$ NMR $\left(600 \mathrm{MHz}, \mathrm{CDCl}_{3}\right) \delta: 0.83-0.88(\mathrm{~m}$, $\left.3 \mathrm{H}, 15-\mathrm{CH}_{3}\right), 0.91-0.95\left(\mathrm{~m}, 3 \mathrm{H}, 8-\mathrm{CH}_{3}\right), 1.09-1.12\left(\mathrm{~m}, 3 \mathrm{H}, 4-\mathrm{CH}_{3}\right), 1.18(\mathrm{~d}$, $\left.J=2.7 \mathrm{~Hz}, 3 \mathrm{H}, 2-\mathrm{CH}_{3}\right), 1.19-1.22\left(\mathrm{~m}, 3 \mathrm{H}, 5^{\prime}-\mathrm{CH}_{3}\right), 1.22-1.24\left(\mathrm{~m}, 3 \mathrm{H}, 10-\mathrm{CH}_{3}\right)$, $1.28\left(\mathrm{~s}, 3 \mathrm{H}, 6-\mathrm{CH}_{3}\right), 1.30-1.34(\mathrm{~m}, 1 \mathrm{H}, \mathrm{H}-7), 1.43-1.45(\mathrm{~m}, 1 \mathrm{H}, \mathrm{H}-7), 1.50$ (s, 3H, 12- $\left.\mathrm{CH}_{3}\right), 1.54-1.58$ (m, 1H, H-14eq), 1.90-1.92 (m, 1H, H-14ax), 1.96 (q, $1 \mathrm{H}, \mathrm{H}-4), 2.21\left(\mathrm{~s}, 6 \mathrm{H},-\mathrm{N}\left(\mathrm{CH}_{3}\right)_{2}\right), 2.23-2.27\left(\mathrm{~m}, 1 \mathrm{H}, \mathrm{H}-3^{\prime}\right), 2.48(\mathrm{q}, 1 \mathrm{H}, \mathrm{H}-$ 10), $2.71\left(\mathrm{~s}, 3 \mathrm{H}, 6-\mathrm{OCH}_{3}\right), 2.87-2.94(\mathrm{~m}, 1 \mathrm{H}, \mathrm{H}-2), 3.11-3.13\left(\mathrm{~m}, 1 \mathrm{H}, \mathrm{H}-2^{\prime}\right)$, 3.25-3.38 (m, 3H, 2'-OH, H-5', 3-O-CH=CH-CH $\left.{ }_{2}-\mathrm{Ar}\right), 3.48(\mathrm{~d}, J=10.3 \mathrm{~Hz}$, $1 \mathrm{H}, \mathrm{H}-3$ ), 3.54 (dd, $J=15.8$ and $7.6 \mathrm{~Hz}, 1 \mathrm{H}, 3-\mathrm{O}-\mathrm{CH}=\mathrm{CH}-\mathrm{CH}_{2}-\mathrm{Ar}$ ), 3.67 (d, $J=2.7 \mathrm{~Hz}, \mathrm{H}-5), 3.75-3.77(\mathrm{~m}, 1 \mathrm{H}, \mathrm{H}-8), 4.23\left(\mathrm{~d}, J=7.5 \mathrm{~Hz}, \mathrm{H}-1^{\prime}\right), 4.53(\mathrm{q}, 1 \mathrm{H}$, $\left.3-\mathrm{O}-\mathrm{CH}=\mathrm{CH}-\mathrm{CH}_{2}-\mathrm{Ar}\right), 4.83(\mathrm{~s}, 1 \mathrm{H}, \mathrm{H}-11), 6.15(\mathrm{~d}, J=6.2 \mathrm{~Hz}, 1 \mathrm{H}, 3-\mathrm{O}-$ $\left.\mathrm{CH}=\mathrm{CH}-\mathrm{CH}_{2}-\mathrm{Ar}\right),[7.16-7.32(\mathrm{~m}, 7 \mathrm{H}), 7.38(\mathrm{~d}, 1 \mathrm{H}, J=7.5 \mathrm{~Hz}), 7.52-7.54(\mathrm{~m}$, $1 \mathrm{H})$, benzyl ring, phenyl]. Including $41 \%$ 3-O-[3-(1'-Phenyl)-E-2-propenyl] isomer, $\delta: 6.71\left(\mathrm{~d}, J=15.8 \mathrm{~Hz}, 1 \mathrm{H}, 3-\mathrm{O}-\mathrm{CH}_{2}-\mathrm{CH}=\mathrm{CH}-\mathrm{Ar}\right), 6.24(\mathrm{dt}, 1 \mathrm{H}, 3-\mathrm{O}-$ $\mathrm{CH}_{2}-\mathrm{CH}=\mathrm{CH}-\mathrm{Ar}$ ).

\section{3-O-[3-(3'-Pyridyl)-Z-1-propenyl]-6-O-methylerythromycin A 9-O-(2-chlorobenzyl)oxime 11,12 -carbonate (9c)}

By a similar prodedure to $\mathbf{9 e}, \mathbf{8 c}$ was prepared as a white solid in $25.6 \%$ yield according to the general procedure for the preparation of $8 \mathbf{e}$, and then deacetylated to give $9 \mathrm{c}(46.7 \%)$. HRMS (ESI) $\left(\mathrm{M}+\mathrm{H}^{+}\right) \mathrm{m} / \mathrm{z} 872.44808$, calcd for $\mathrm{C}_{46} \mathrm{H}_{67} \mathrm{ClN}_{3} \mathrm{O}_{11}$ 872.44586. ${ }^{1} \mathrm{H} \mathrm{NMR}\left(400 \mathrm{MHz}, \mathrm{CDCl}_{3}\right) \delta: 0.84$ (t, 3H, 15$\left.\mathrm{CH}_{3}\right), 0.92\left(\mathrm{~d}, 3 \mathrm{H}, 8-\mathrm{CH}_{3}\right), 1.15-1.13\left(\mathrm{~m}, 6 \mathrm{H}, 4-\mathrm{CH}_{3}, 2-\mathrm{CH}_{3}\right), 1.16(\mathrm{~d}$, $\left.J=6.1 \mathrm{~Hz}, 3 \mathrm{H}, 5^{\prime}-\mathrm{CH}_{3}\right), 1.21\left(\mathrm{~d}, J=6.8 \mathrm{~Hz}, 3 \mathrm{H}, 10-\mathrm{CH}_{3}\right), 1.25\left(\mathrm{~s}, 3 \mathrm{H}, 6-\mathrm{CH}_{3}\right)$, $1.27-1.30(\mathrm{~m}, 1 \mathrm{H}, \mathrm{H}-7), 1.41-1.45(\mathrm{~m}, 1 \mathrm{H}, \mathrm{H}-7), 1.48\left(\mathrm{~s}, 3 \mathrm{H}, 12-\mathrm{CH}_{3}\right), 1.51-$ 1.59 (m, 2H, H-14ax, H-4'), 1.86-1.97 (m, 2H, H-14eq, H-4), 2.23 (s, 6H, $\left.\mathrm{N}\left(\mathrm{CH}_{3}\right)_{2}\right), 2.28\left(\mathrm{~m}, 1 \mathrm{H}, \mathrm{H}-3^{\prime}\right), 2.46(\mathrm{q}, 1 \mathrm{H}, \mathrm{H}-10), 2.70\left(\mathrm{~s}, 3 \mathrm{H}, 6-\mathrm{OCH}_{3}\right), 2.87-$ $2.94(\mathrm{~m}, 1 \mathrm{H}, \mathrm{H}-2), 3.13\left(\mathrm{dd}, J=7.3\right.$ and $\left.10.1 \mathrm{~Hz}, 1 \mathrm{H}, \mathrm{H}-2^{\prime}\right), 3.28-3.33(\mathrm{~m}, 3 \mathrm{H}$, $\left.\mathrm{H}-5^{\prime}, 2^{\prime}-\mathrm{OH}, 3-\mathrm{O}-\mathrm{CH}=\mathrm{CH}-\mathrm{CH}_{2}-\mathrm{Ar}\right), 3.47-3.55(\mathrm{~m}, 2 \mathrm{H}, \mathrm{H}-3,3-\mathrm{O}-\mathrm{CH}=\mathrm{CH}-$ $\left.\mathrm{CH}_{2}-\mathrm{Ar}\right), 3.64(\mathrm{~d}, J=2.7 \mathrm{~Hz}, 1 \mathrm{H}, \mathrm{H}-5), 3.74-3.82(\mathrm{~m}, 1 \mathrm{H}, \mathrm{H}-8), 4.19(\mathrm{~d}$, $\left.J=7.2 \mathrm{~Hz}, 1 \mathrm{H}, \mathrm{H}-\mathrm{I}^{\prime}\right), 4.48-4.54\left(\mathrm{q}, 1 \mathrm{H}, 3-\mathrm{O}-\mathrm{CH}=\mathrm{CH}-\mathrm{CH}_{2}-\mathrm{Ar}\right), 4.82(\mathrm{~s}, 1 \mathrm{H}$, $\mathrm{H}-11), 5.24-5.09\left(\mathrm{~m}, 3 \mathrm{H},-\mathrm{CH}_{2}-\mathrm{PhCl}, \mathrm{H}-13\right), 6.18(\mathrm{~d}, J=6.0 \mathrm{~Hz}, 1 \mathrm{H}, 3-\mathrm{O}-$ $\left.\mathrm{CH}=\mathrm{CH}-\mathrm{CH}_{2}-\mathrm{Ar}\right),[7.16-7.26(\mathrm{~m}, 3 \mathrm{H}), 7.30(\mathrm{dd}, J=1.2$ and $7.7 \mathrm{~Hz}, 1 \mathrm{H})$,
7.50-7.52 (m, 2H), 8.41-8.47 (m, 2H), benzyl ring, pyridyl]. ${ }^{13} \mathrm{C} \operatorname{NMR}\left(100 \mathrm{MHz}, \mathrm{CDCl}_{3}\right) \delta: 174.3$ (C-1), 165.1 (C-9), 154.6 (carbonate), $149.7,148.5,147.3,136.6,136.0,135.6,133.5,130.9,128.9,128.6,126.4,123.2$, 101.9, 101.6, 89.5, 84.8, 82.8, 79.7, 78.4, 75.4, 72.6, 70.3, 69.1, 65.6, 49.3, 44.5, $40.2\left(-\mathrm{N}\left(\mathrm{CH}_{3}\right)_{2}\right), 37.1,36.4,32.8,27.7,25.8,22.1,21.1,19.3,18.7,15.6,15.4$, $12.8,10.0,8.8$

\section{3-O-allyl-6-O-methylerythromycin A 9-O-(2-chlorobenzyl)oxime} 11,12-carbonate $(9 a)$

Compound 7 was heated to reflux in $\mathrm{MeOH}$ for $3 \mathrm{~h}$. After the solvent was evaporated, the crude product was purified by column chromatography (silica gel, 15:0.3:0.1 $\mathrm{CH}_{2} \mathrm{Cl}_{2} / \mathrm{C}_{2} \mathrm{H}_{5} \mathrm{OH} / \mathrm{NH}_{3} \cdot \mathrm{H}_{2} \mathrm{O}$ ) to give $9 \mathrm{a}$ in $62.1 \%$ yield. HRMS (ESI) $\left(\mathrm{M}+\mathrm{H}^{+}\right) \mathrm{m} / z$ 795.42088, calcd for $\mathrm{C}_{41} \mathrm{H}_{64} \mathrm{ClN}_{2} \mathrm{O}_{11}$ 795.41932. ${ }^{1} \mathrm{H}$ NMR $\left(400 \mathrm{MHz}, \mathrm{CDCl}_{3}\right) \delta: 0.86\left(\mathrm{t}, 3 \mathrm{H}, 15-\mathrm{CH}_{3}\right), 0.92\left(\mathrm{~d}, J=7.0 \mathrm{~Hz}, 3 \mathrm{H}, 8-\mathrm{CH}_{3}\right), 1.03$ (d, $\left.J=7.5 \mathrm{~Hz}, 3 \mathrm{H}, 4-\mathrm{CH}_{3}\right), 1.17-1.23\left(\mathrm{~m}, 6 \mathrm{H}, 10-\mathrm{CH}_{3}, 5^{\prime}-\mathrm{CH}_{3}\right), 1.25-1.29$ (m, 6H, 2- $\left.\mathrm{CH}_{3}, 6-\mathrm{CH}_{3}\right), 1.32-1.35$ (m, 1H, H-7), 1.41-1.44 (m, 1H, H-7), 1.48 (s, 3H, 12- $\left.\mathrm{CH}_{3}\right), 1.51-1.67$ (m, 2-3H, H-14ax, H-4'), 1.86-1.92 (m, 2H, H-4, $\mathrm{H}-14 \mathrm{eq}), 2.28$ (s, 6H, $\left.-\mathrm{N}\left(\mathrm{CH}_{3}\right)_{2}\right), 2.46-2.50\left(\mathrm{~m}, 2 \mathrm{H}, \mathrm{H}-3^{\prime}, \mathrm{H}-10\right), 2.72(\mathrm{~s}, 3 \mathrm{H}$, 6- $\left.\mathrm{CH}_{3}\right), 2.80-2.89(\mathrm{~m}, 1 \mathrm{H}, \mathrm{H}-2), 3.16\left(\mathrm{dd}, J=7.2\right.$ and $\left.10.1 \mathrm{~Hz}, 1 \mathrm{H}, \mathrm{H}-2^{\prime}\right), 3.21-$ $3.24\left(\mathrm{~m}, 2 \mathrm{H}, \mathrm{H}-3,2^{\prime}-\mathrm{OH}\right), 3.42-3.46\left(\mathrm{~m}, 1 \mathrm{H}, \mathrm{H}-5^{\prime}\right), 3.70(\mathrm{~d}, J=3.2 \mathrm{~Hz}, 1 \mathrm{H}, \mathrm{H}-$ 5), 3.74-3.78 (m, $1 \mathrm{H}, \mathrm{H}-8), 4.05-4.30\left(\mathrm{~m}, 2 \mathrm{H},-\mathrm{CH}_{2}-\mathrm{CH}=\mathrm{CH}_{2}\right), 4.37(\mathrm{~d}$, $J=7.2 \mathrm{~Hz}, 1 \mathrm{H}, \mathrm{H}-\mathrm{l}^{\prime}$ ), 4.81 (s, 1H, H-11), 5.11-5.26 (m, $5 \mathrm{H}, \mathrm{H}-13,-\mathrm{CH}_{2}-\mathrm{PhCl}$, $\left.-\mathrm{CH}=\mathrm{CH}_{2}\right), 5.35\left(\mathrm{dd}, J=1.6\right.$ and $\left.17.2 \mathrm{~Hz}, 1 \mathrm{H},-\mathrm{CH}=\mathrm{CH}_{2}\right) 5.84-5.93(\mathrm{~m}, 1 \mathrm{H},-$ $\left.\mathrm{CH}=\mathrm{CH}_{2}\right),[7.17-7.26(\mathrm{~m}, 2 \mathrm{H}), 7.32(\mathrm{dd}, J=1.2$ and $7.8 \mathrm{~Hz}, 1 \mathrm{H}), 7.53(\mathrm{dd}$, $J=1.6$ and $7.5 \mathrm{~Hz}, 1 \mathrm{H})$, benzyl ring]

\section{3-O-allyl-6-O-methylerythromycin A 9-O-(2-chlorobenzyl)oxime} (12a)

Compound $12 \mathrm{a}$ was synthesized by a similar procedure to $\mathbf{9 a}$ in $70.0 \%$ yield. MS(MALDI-TOF) $\left(\mathrm{M}+\mathrm{Na}^{+}\right) \mathrm{m} / z$ 791.2, calcd for $\mathrm{C}_{40} \mathrm{H}_{65} \mathrm{ClN}_{2} \mathrm{O}_{10} 768.4(\mathrm{M})$. ${ }^{1} \mathrm{H}$ NMR $\left(400 \mathrm{MHz}, \mathrm{CDCl}_{3}\right) \delta: 0.82\left(\mathrm{t}, 3 \mathrm{H}, 15-\mathrm{CH}_{3}\right), 0.96\left(\mathrm{~d}, 3 \mathrm{H}, 8-\mathrm{CH}_{3}\right), 1.05$ $\left(\mathrm{d}, 3 \mathrm{H}, 4-\mathrm{CH}_{3}\right), 1.12\left(\mathrm{~d}, 3 \mathrm{H}, 10-\mathrm{CH}_{3}\right), 1.14\left(\mathrm{~s}, 3 \mathrm{H}, 12-\mathrm{CH}_{3}\right), 1.20(\mathrm{~d}, 3 \mathrm{H}$, $\left.5^{\prime}-\mathrm{CH}_{3}\right), 1.25\left(\mathrm{~d}, 3 \mathrm{H}, 2-\mathrm{CH}_{3}\right), 1.31\left(\mathrm{~s}, 3 \mathrm{H}, 6-\mathrm{CH}_{3}\right), 1.39-1.52(\mathrm{~m}, 3 \mathrm{H}, \mathrm{H}-7$, H-14ax), 1.62-1.66 (m, 1H, H-4'), 1.92-2.08 (m, 2H, H-4, H-14eq), 2.27 $\left(\mathrm{s}, 6 \mathrm{H},-\mathrm{N}\left(\mathrm{CH}_{3}\right)_{2}\right), 2.45-2.46\left(\mathrm{~m}, 1 \mathrm{H}, \mathrm{H}-3^{\prime}\right), 2.57(\mathrm{q}, 1 \mathrm{H}, \mathrm{H}-10), 2.81-2.84$ (m, 1H, H-2), $2.85\left(\mathrm{~s}, 3 \mathrm{H}, 6-\mathrm{CH}_{3}\right), 3.14-3.27\left(\mathrm{~m}, 4 \mathrm{H}, \mathrm{H}-2^{\prime}, \mathrm{H}-3,12-\mathrm{OH}\right.$, $2^{\prime}-\mathrm{OH}$ ), 3.42-3.46 ( $\left.\mathrm{m}, 1 \mathrm{H}, \mathrm{H}-5^{\prime}\right), 3.74-3.76$ ( $\left.\mathrm{m}, \mathrm{H}-5, \mathrm{H}-8, \mathrm{H}-11\right), 4.09-4.28$ $\left(\mathrm{m}, 2 \mathrm{H},-\mathrm{CH}_{2}-\mathrm{CH}=\mathrm{CH}_{2}\right), 4.39(\mathrm{~s}, 1 \mathrm{H}, 11-\mathrm{OH}), 4.42\left(\mathrm{~d}, 1 \mathrm{H}, \mathrm{H}-1^{\prime}\right), 5.07-5.37$ $\left(\mathrm{m}, 5 \mathrm{H}, \mathrm{H}-13,-\mathrm{CH}_{2}-\mathrm{PhCl},-\mathrm{CH}=\mathrm{CH}_{2}\right), 5.86-5.93\left(\mathrm{~m}, 1 \mathrm{H},-\mathrm{CH}=\mathrm{CH}_{2}\right), 7.19-$ $7.40\left(\mathrm{~m}, 4 \mathrm{H}\right.$, benzyl ring]. ${ }^{13} \mathrm{C} \mathrm{NMR}\left(100 \mathrm{MHz}, \mathrm{CDCl}_{3}\right) \delta: 175.0,170.8,135.8$, 134.7, 133.2, 129.6, 129.2, 128.7, 126.5, 116.0, 101.2, 84.4, 79.2, 78.3, 76.8, 74.2, $73.9,72.6,70.7,70.3,68.9,65.3,49.9,44.6,40.2,36.9,33.0,28.8,26.4,21.3$, $21.1,19.4,18.5,16.0,15.3,15.2,10.4,8.6$.

\section{3-O-[3-(3'-Pyridyl)-Z-1-propenyl]-6-O-methylerythromycin A 9-O-(2-chlorobenzyl)oxime (12c)}

Compound $12 \mathrm{c}$ was synthesized by a similar procedure to $9 \mathbf{c}$ ( $26.3 \%$ in two steps). HRMS (ESI) $\left(\mathrm{M}+\mathrm{H}^{+}\right) \mathrm{m} / \mathrm{z} 846.4648$, calcd for $\mathrm{C}_{45} \mathrm{H}_{69} \mathrm{ClN}_{3} \mathrm{O}_{10} 846.4666$. ${ }^{1} \mathrm{H} \mathrm{NMR}\left(400 \mathrm{MHz}, \mathrm{CDCl}_{3}\right) \delta: 0.81\left(\mathrm{t}, 3 \mathrm{H}, 15-\mathrm{CH}_{3}\right), 0.96\left(\mathrm{~d}, 3 \mathrm{H}, 8-\mathrm{CH}_{3}\right), 1.11-$ $1.24\left(\mathrm{~m}, 16 \mathrm{H}, 4-\mathrm{CH}_{3}, 2-\mathrm{CH}_{3}, 10-\mathrm{CH}_{3}, 5^{\prime}-\mathrm{CH}_{3}, 12-\mathrm{CH}_{3}, \mathrm{H}-4^{\prime} \mathrm{ax}\right), 1.30(\mathrm{~s}, 3 \mathrm{H}$, 6- $\mathrm{CH}_{3}$ ), 1.30-2.12 (m, 6H, H-7, H-14ax, H-4'eq, H-14eq, H-4), 2.30 (s, 6H, $\left.\mathrm{N}\left(\mathrm{CH}_{3}\right)_{2}\right), 2.31-2.34\left(\mathrm{~m}, 1 \mathrm{H}, \mathrm{H}-3^{\prime}\right), 2.56(\mathrm{q}, 1 \mathrm{H}, \mathrm{H}-10), 2.87\left(\mathrm{~s}, 3 \mathrm{H}, 6-\mathrm{OCH}_{3}\right)$, 2.88-2.91 (m, 1H, H-2), 3.31-3.54 (m, 7H, H-5', H-2', 12-OH, 2'-OH, 3-O$\left.\mathrm{CH}=\mathrm{CH}-\mathrm{CH}_{2}-\mathrm{Ar}, \mathrm{H}-3\right), 3.67(\mathrm{~m}, 3 \mathrm{H}, \mathrm{H}-5, \mathrm{H}-8, \mathrm{H}-11), 4.22(\mathrm{~d}, J=7.3 \mathrm{~Hz}, 1 \mathrm{H}$, $\left.\mathrm{H}-1^{\prime}\right), 4.42(\mathrm{~s}, 1 \mathrm{H}, 11-\mathrm{OH}), 4.50\left(\mathrm{q}, 1 \mathrm{H}, 3-\mathrm{O}-\mathrm{CH}=\mathrm{CH}-\mathrm{CH}_{2}-\mathrm{Ar}\right), 5.06-5.21$ $\left(\mathrm{m}, 3 \mathrm{H},-\mathrm{CH}_{2}-\mathrm{PhCl}, \mathrm{H}-13\right), 6.20\left(\mathrm{~d}, J=6.1 \mathrm{~Hz}, 1 \mathrm{H}, 3-\mathrm{O}-\mathrm{CH}=\mathrm{CH}-\mathrm{CH}_{2}-\mathrm{Ar}\right)$, $7.20-8.49(\mathrm{~m}, 8 \mathrm{H}$, benzyl ring, pyridyl).

\section{3-O-[3-(5'-Pyrimidyl)-Z-1-propenyl]-6-O-methylerythromycin A 9-O-(2-chlorobenzyl)oxime (12d)}

Compound 12d was synthesized by a similar procedure to $9 \mathrm{~d}$ (12.2\% in two steps). HRMS (ESI) $\left(\mathrm{M}+\mathrm{H}^{+}\right) \mathrm{m} / \mathrm{z} 847.4612$, calcd for $\mathrm{C}_{44} \mathrm{H}_{68} \mathrm{ClN}_{4} \mathrm{O}_{10} 847.4618$. ${ }^{1} \mathrm{H}$ NMR $\left(400 \mathrm{MHz}, \mathrm{CDCl}_{3}\right), \delta: 0.81\left(\mathrm{t}, 3 \mathrm{H}, 15-\mathrm{CH}_{3}\right), 0.97$ (d, $3 \mathrm{H}, 8-\mathrm{CH}_{3}$ ), 1.11-1.24 (m, 16H, $\left.4-\mathrm{CH}_{3}, 2-\mathrm{CH}_{3}, 10-\mathrm{CH}_{3}, 5^{\prime}-\mathrm{CH}_{3}, 12-\mathrm{CH}_{3}, \mathrm{H}-4^{\prime} \mathrm{ax}\right), 1.27$ (s, 3H, 6- $\left.\mathrm{CH}_{3}\right), 1.30-2.20$ (m, 6H, H-7, H-14ax, H-4'eq, H-14eq, H-4), 2.38 
(s, 6H, $\left.-\mathrm{N}\left(\mathrm{CH}_{3}\right)_{2}\right), 2.46\left(\mathrm{~m}, \mathrm{H}-3^{\prime}\right), 2.56(\mathrm{q}, 1 \mathrm{H}, \mathrm{H}-10), 2.87$ (s, 3H, 6- $\left.\mathrm{OCH}_{3}\right)$, 2.88-2.91 (m, 1H, H-2), 3.23-3.54 (m, 7H, H-5', H-2', 12-OH, 2'-OH, 3-O$\left.\mathrm{CH}=\mathrm{CH}-\mathrm{CH}_{2}-\mathrm{Ar}, \mathrm{H}-8\right), 3.67(\mathrm{~d}, J=2.8 \mathrm{~Hz}, 1 \mathrm{H}, \mathrm{H}-5), 3.74-3.76(\mathrm{~m}, 2 \mathrm{H}, \mathrm{H}-3$, $\mathrm{H}-11), 4.20$ (d, $\left.J=7.2 \mathrm{~Hz}, 1 \mathrm{H}, \mathrm{H}-1^{\prime}\right), 4.41(\mathrm{~s}, 1 \mathrm{H}, 11-\mathrm{OH}), 4.50$ (q, $1 \mathrm{H}, 3-\mathrm{O}-$ $\left.\mathrm{CH}=\mathrm{CH}-\mathrm{CH}_{2}-\mathrm{Ar}\right), 5.07-5.20\left(\mathrm{~m}, 3 \mathrm{H}, \mathrm{H}-13,-\mathrm{CH}_{2}-\mathrm{PhCl}\right), 6.25(\mathrm{~d}, J=6.0 \mathrm{~Hz}$, $\left.1 \mathrm{H}, 3-\mathrm{O}-\mathrm{CH}=\mathrm{CH}-\mathrm{CH}_{2}-\mathrm{Ar}\right), 7.19-7.41(\mathrm{~m}, 4 \mathrm{H}$, benzyl ring], [8.62 (s, $2 \mathrm{H})$, $9.06(\mathrm{~s}, 1 \mathrm{H})$, pyrimidyl].

\section{3-O-[3-(3'-Quinolyl)-Z-1-propenyl]-6-O-methylerythromycin A 9-O-(2-chlorobenzyl)oxime (12e)}

Compound $12 \mathbf{e}$ was synthesized by a similar procedure to $9 \mathbf{e}(45.0 \%$ in two steps). HRMS (ESI) $\left(\mathrm{M}+\mathrm{H}^{+}\right) \mathrm{m} / \mathrm{z} 896.4802$, calcd for $\mathrm{C}_{49} \mathrm{H}_{71} \mathrm{ClN}_{3} \mathrm{O}_{10} 896.4822$. ${ }^{1} \mathrm{H}$ NMR $\left(400 \mathrm{MHz}, \mathrm{CDCl}_{3}\right) \delta: 0.82\left(\mathrm{t}, 3 \mathrm{H}, 15-\mathrm{CH}_{3}\right), 0.97\left(\mathrm{~d}, 3 \mathrm{H}, 8-\mathrm{CH}_{3}\right)$, 1.11-1.27 (m, $\left.16 \mathrm{H}, 4-\mathrm{CH}_{3}, 2-\mathrm{CH}_{3}, 10-\mathrm{CH}_{3}, 5^{\prime}-\mathrm{CH}_{3}, 12-\mathrm{CH}_{3}, \mathrm{H}-4^{\prime} \mathrm{ax}\right), 1.30$ (s, $3 \mathrm{H}, 6-\mathrm{CH}_{3}$ ), 1.30-2.20 (m, 6H, H-7, H-14ax, H-4'eq, H-14eq, H-4), 2.22-2.32 $\left(\mathrm{m}, 7 \mathrm{H},-\mathrm{N}\left(\mathrm{CH}_{3}\right)_{2}, \mathrm{H}-3^{\prime}\right), 2.57$ (q, $\left.1 \mathrm{H}, \mathrm{H}-10\right), 2.89\left(\mathrm{~s}, 3 \mathrm{H}, 6-\mathrm{OCH}_{3}\right), 2.89-2.96$ (m, 1H, H-2), 3.17 (dd, 1H, H-2'), $3.26(\mathrm{~s}, 1 \mathrm{H}, 12-\mathrm{OH}), 3.28-3.55(\mathrm{~m}, 4 \mathrm{H}, \mathrm{H}-$ $\left.5^{\prime}, 3-\mathrm{O}-\mathrm{CH}=\mathrm{CH}-\mathrm{CH}_{2}-\mathrm{Ar}, \mathrm{H}-3\right), 3.71-3.78(\mathrm{~m}, 3 \mathrm{H}, \mathrm{H}-5, \mathrm{H}-11, \mathrm{H}-8), 4.24(\mathrm{~d}$ $\left.J=7.4 \mathrm{~Hz}, 1 \mathrm{H}, \mathrm{H}_{-1} \mathrm{l}^{\prime}\right), 4.43(\mathrm{~s}, 1 \mathrm{H}, 11-\mathrm{OH}), 4.59\left(\mathrm{q}, 1 \mathrm{H}, 3-\mathrm{O}-\mathrm{CH}=\mathrm{CH}-\mathrm{CH}_{2-}\right.$ Ar), 5.07-5.22 (m, $\left.3 \mathrm{H},-\mathrm{CH}_{2}-\mathrm{PhCl}, \mathrm{H}-13\right), 6.27(\mathrm{~d}, J=6.1 \mathrm{~Hz}, 1 \mathrm{H}, 3-\mathrm{O}-$ $\left.\mathrm{CH}=\mathrm{CH}-\mathrm{CH}_{2}-\mathrm{Ar}\right), \quad 7.21-7.42 \quad(\mathrm{~m}, 4 \mathrm{H}$, benzyl ring $), 7.53-8.83(\mathrm{~m}, 6 \mathrm{H}$, quinolyl).

\section{3-O-[3-(4'-Isoquinolyl)-Z-1-propenyl]-6-O-methylerythromycin A 9-O-(2-chlorobenzyl)oxime (12f)}

Compound $12 \mathrm{f}$ was synthesized by a similar procedure to $9 \mathrm{f}(26.2 \%$ in two steps). HRMS (ESI) $\left(\mathrm{M}+\mathrm{H}^{+}\right) \mathrm{m} / \mathrm{z} 896.4819$, calcd for $\mathrm{C}_{49} \mathrm{H}_{71} \mathrm{ClN}_{3} \mathrm{O}_{10} 896.4822$. ${ }^{1} \mathrm{H}$ NMR $\left(400 \mathrm{MHz}, \mathrm{CDCl}_{3}\right) \delta: 0.83\left(\mathrm{t}, 3 \mathrm{H}, 15-\mathrm{CH}_{3}\right), 0.93(\mathrm{~d}, J=6.9 \mathrm{~Hz}, 3 \mathrm{H}$, $\left.8-\mathrm{CH}_{3}\right), 0.97\left(\mathrm{~d}, 3 \mathrm{H}, 8-\mathrm{CH}_{3}\right), 1.11-1.27\left(\mathrm{~m}, 16 \mathrm{H}, 4-\mathrm{CH}_{3}, 2-\mathrm{CH}_{3}, 10-\mathrm{CH}_{3}\right.$, $\left.5^{\prime}-\mathrm{CH}_{3}, 12-\mathrm{CH}_{3}, \mathrm{H}-4^{\prime} \mathrm{ax}\right), 1.32\left(\mathrm{~s}, 3 \mathrm{H}, 6-\mathrm{CH}_{3}\right), 1.30-2.20(\mathrm{~m}, 6 \mathrm{H}, \mathrm{H}-7, \mathrm{H}-14 \mathrm{ax}$, H-4'eq, H-14eq, H-4), 2.23 (s, 6H, $\left.-\mathrm{N}\left(\mathrm{CH}_{3}\right)_{2}\right), 2.25-2.26$ (m, $\left.1 \mathrm{H}, \mathrm{H}-3^{\prime}\right), 2.58$ (q, 1H, H-10), 2.89 (s, 3H, 6- $\left.\mathrm{OCH}_{3}\right), 2.97-2.99(\mathrm{~m}, 1 \mathrm{H}, \mathrm{H}-2), 3.15-3.27(\mathrm{~m}$, $\left.4 \mathrm{H}, \mathrm{H}-2^{\prime}, \mathrm{H}-5^{\prime}, 12-\mathrm{OH}, 2^{\prime}-\mathrm{OH}\right), 3.57(\mathrm{~d}, J=10.2 \mathrm{~Hz}, 1 \mathrm{H}, \mathrm{H}-3), 3.58-3.98(\mathrm{~m}$, $\left.5 \mathrm{H}, \mathrm{H}-11, \mathrm{H}-5, \mathrm{H}-8,3-\mathrm{O}-\mathrm{CH}=\mathrm{CH}-\mathrm{CH}_{2}-\mathrm{Ar}\right), 4.29$ (d, J=7.2 Hz, $\left.1 \mathrm{H}, \mathrm{H}-\mathrm{I}^{\prime}\right)$, $4.41(\mathrm{~s}, 1 \mathrm{H}, 11-\mathrm{OH}), 4.57\left(\mathrm{q}, 1 \mathrm{H}, 3-\mathrm{O}-\mathrm{CH}=\mathrm{CH}-\mathrm{CH}_{2}-\mathrm{Ar}\right), 5.07-5.23(\mathrm{~m}, 3 \mathrm{H}$, $\left.-\mathrm{CH}_{2}-\mathrm{PhCl}, \mathrm{H}-13\right), 6.22\left(\mathrm{~d}, \mathrm{~J}=6.1 \mathrm{~Hz}, 1 \mathrm{H}, 3-\mathrm{O}-\mathrm{CH}=\mathrm{CH}-\mathrm{CH}_{2}-\mathrm{Ar}\right.$ ), $7.21-7.42$ ( $\mathrm{m}, 4 \mathrm{H}$, benzyl ring), 7.61-9.14 ( $\mathrm{m}, 6 \mathrm{H}$, isoquinolyl).

Crystallographic data for the structure of 9 f in this paper have been deposited with the Cambridge Crystallographic Data Centre as supplementary publication number CCDC 729701. Copies of the data can be obtained, free of charge, on application to CCDC, 12 Union Road, Cambridge CB2 1EZ, UK [fax: +44 (0) 1223-336033 or E-mail: deposit@ccdc.cam.ac.uk].

\section{ACKNOWLEDGEMENTS}

This research was supported by the National Natural Science Foundation of China (20602002). We thank Prof. Xiang Hao and Ms. Tong-Ling Liang,
Institute of Chemistry Chinese Academy of Sciences, for single X-ray crystal structure determination.

1 Kurath, P., Jones, P. H., Egan, R. S. \& Perun, T. J. Acid degradation of erythromycin A and erythromycin B. Experientia 27, 362 (1971).

2 Watanabe, Y., Morimoto, S., Adachi, T., Kashimura, M. \& Asaka, T. Chemical modification of erythromycins. 9. Selective methylation the C-6 hydroxyl group of erythromycin A oxime derivatives and preparation of clarithromycin. J. Antibiot. 46, 647-660 (1993).

3 Plata, D. J. et al. The synthesis of ketolide antibiotic ABT-773 (cethromycin). Tetrahedron 60, 10171-10180 (2004).

$4 \mathrm{Ma}$, Z. et al. Novel erythromycin derivatives with aryl groups tethered to the C-6 position are potent protein synthesis inhibitors and active against multidrug-resistant respiratory pathogens. J. Med. Chem. 44, 4137-4156 (2001).

5 Clark, R. F. et al. Synthesis and antibacterial activity of novel 6-0-substituted erythromycin A derivatives.. Bioorg. Med. Chem. Lett. 10, 815-819 (2000).

6 Agouridas, C. et al. Synthesis and antibacterial activity of ketolides (6-0-methyl-3oxoerythromycin derivatives): a new class of antibacterials highly potent against macrolide-resistant and -susceptible respiratory pathogens. J. Med. Chem. 41, 4080-4100 (1998)

7 Tanikawa, T. et al. Synthesis and antibacterial activity of acylides (3-0-acyl-erythromycin derivatives): a novel class of macrolide antibiotics. J. Med. Chem. 44, 4027-4030 (2001).

8 Tang, D. et al. Design, synthesis, and antibacterial activities of novel 3,6-bicyclolide oximes: length optimization and zero carbon linker oximes. Bioorg. Med. Chem. Lett. 18, 5078-5082 (2008)

9 Elliott, R. L. et al. Anhydrolide macrolides. 1. Synthesis and antibacterial activity of 2,3-anhydro-6-0-methyl 11,12-carbamate erythromycin A analogues. J. Med. Chem. 41, 1651-1659 (1998).

10 Denis, A. et al. Beta-keto-ester chemistry and ketolides. Synthesis and antibacterial activity of 2-halogeno, 2-methyl and 2,3 enol-ether ketolides. Bioorg. Med. Chem. Lett. 10, 2019-2022 (2000).

11 Elliott, R. L. et al. Novel 3-deoxy-3-descladinosyl-6-0-methyl erythromycin A analogues. Synthesis and in vitro activity. Bioorg. Med. Chem. Lett. 7, 641-646 (1997).

12 Misawa, Y., Asaka, T., Kashimura, M., Morimoto, S. \& Hatayama, K. 5-0-desosaminylerythronolide derivative EP 682, 03824 January (1994).

13 Cheng, H. M. et al. Synthesis and SAR of azalide 3,6-ketal aromatic derivatives as potent gram-positive and gram-negative antibacterial agents. Bioorg. Med. Chem. Lett. 12, 2431-2434 (2002).

14 Heggelund, A. \& Undheim, K. Descladinosyl erythromycin in phosgene-assisted cyclic 3,6-ether formation. Tetrahedron Lett. 49, 5569-5571 (2008).

15 Douthwaite, S., Hansen, L. H. \& Mauwais, P. Macrolide-ketolide inhibition of MLSresistant ribosomes is improved by alternative drug interaction with domain II of 23S rRNA. Mol. Microbiol. 36, 183-192 (2000).

16 Furuuchi, T. et al. Design and synthesis of novel leucomycin analogues modified at the C-3 position. Part II: 3-0-(3-Aryl-2-propenyl)leucomycin analogues. Bioorg. Med. Chem. 16, 4401-4418 (2008).

17 Watanabe, Y. et al. Chemical modification of erythromycins. 12. A facile synthesis of clarithromycin (6-0-methylerythromycin A) via 2'-silylethers of erythromycin A derivatives. J. Antibiot 46, 1163-1167 (1993).

18 Clinical and Laboratory Standards Institute. Methods for Dilution Antimicrobial Susceptibility Tests for Bacteria that Grow Aerobically. Approved Standard M7-A7, Seventh Edition, Clinical and Laboratory Standards Institute, Wayne, PA, (2006) 